\title{
Thermo-mechanical modelling of rock-like materials at very high temperature: application to ceramic refractories
}

\author{
Alessandro Gajo ${ }^{*} \dagger$ and Francesco Cecinato ${ }^{*} \ddagger$ \\ *DICAM, University of Trento, Via Mesiano 77 - 38123 Trento, Italy \\ †Email: alessandro.gajo@unitn.it \\ ${ }_{\ddagger}$ Corresponding Author. Email: francesco.cecinato@unitn.it
}

\begin{abstract}
Rock-like materials like ceramic refractories, in working conditions may be subject to large temperature variations. To simulate practical applications, bespoke constituive modelling is required. In this work a general, thermodynamically consistent framework, able to incorporate key micromechanical features of the material behaviour, and applicable to a wide range of geomaterials, is formulated and validated. Different thermodynamic potentials are proposed to deal with both reversibility and irreversibility. A key advantage of this approach is the ability to freely choose the thermal dependency interpolation functions. Extensive model validation is provided by correctly reproducing both reversible and irreversible experimental trends of different materials under different loading conditions. It is found that even for simple materials, if a sample is subject to a large stress level, its thermal and mechanical responses become unexpectedly coupled. The proposed modelling framework is not limited to refractories and can be easily adapted to different types of rock-like materials.
\end{abstract}

Keywords: elasticity, elasto-plasticity, high temperature, refractories, porous media

\section{Introduction}

Several applications in manufacturing engineering imply the exposure of rocklike materials, like refractories, to very high temperature values, and possibly 
also large temperature gradients. Although several experimental programs have been carried out to measure relevant properties of ceramic refractories at high temperature, and some models (e.g., [27], [1], [25], [17]) have been proposed to reproduce the main aspects of their thermo-mechanical behaviour, few attempts have been made to date to propose a general framework, able to incorporate key micromechanical features of the thermo-elasto-plastic material behaviour, with a thermodynamically consistent formulation, applicable to a wide range of different materials. Due to data availability, special focus in this work is given to ceramic materials used in the steel production industry. Based on their different behaviour upon thermal cycling, the two categories of (i) single component materials (like alumina) and (ii) casted or pressed composite refractories are identified, and their constitutive peculiarities are modelled with bespoke mechanisms.

Ceramic refractories are usually very stiff (with elastic stiffness ranging between 10 and $200 \mathrm{GPa}$ ) and characterised by thermal expansion coefficients usually ranging between $3 \times$ and $6 \times 10^{-6} \mathrm{~K}^{-1}$ (e.g., see [11], [32], [10]). When subjected to temperature variations of nearly $1500 \mathrm{~K}$, the associated thermal strain is consequently very large $(0.45-0.90 \%)$ which, if prevented, would lead to huge induced stresses $(45-1800 \mathrm{MPa})$. Although refractory materials, to allow for their inevitable, thermally induced dilative deformation, are not usually constrained at the external boundaries, thermal gradients to which refractory pieces are subject in working conditions (e.g. in steel making processes) may be very large, thus possibly inducing large self-equilibrated internal stresses. Furthermore, certain refractory materials typically exhibit irreversibility upon temperature cycling, even at low applied stresses, which may lead to accumulated permanent changes (i.e. degradation) of their elastic and/or strength properties during their working life. Hence, simulating accurately the thermo-mechanical behaviour of these materials appears of paramount importance.

Thermo-mechanical and thermo-physical properties of refractories typically exhibit a wide variation among different materials, mainly depending upon their chemical composition, forming process and curing temperature. It has emerged from a comprehensive literature review [8] that while the specific heat capacity $c_{p}$ (or $c_{v}$ ) and thermal expansion coefficient $\alpha$ exhibit characteristic trends of thermal variation that could be considered common to most ceramic material types, the modulus of elasticity $E$ shows a much wider variety of trends with temperature. It appears that, somewhat counter to engineering intuition, the elastic modulus of certain refractories (both measured with dynamic techniques or by means of mechanical, pseudo-static testing) does not exhibit a steady decrease with increasing temperature, but an initial horizontal or slightly decreasing trend, followed by a marked nonlinear increase, sometimes followed by an abrupt drop beyond a threshold temperature (e.g., see [17], [32], [16], [10]). Moreover, while single component refractory materials exhibit a reversible thermal behaviour (thus if heated at very high temperatures and then cooled, they do not show any permanent change of their thermo-mechanical properties), composite materials ex- 
hibit hysteresis when subjected to a heating/cooling cycle (even at low applied stresses).

Overall, when measured in unconfined conditions, a general tendency is observed for both $\alpha$ and $c_{p}$ to increase with increasing temperature, with a more or less marked tendency to reach a horizontal asymptote at large temperature values. This applies to both single-component materials such as alumina or glassy carbon (e.g. [32]) and composite materials, such as carbon-bonded refractories (e.g. [11]). In contrast, elastic stiffness exhibits a decreasing and mostly reversible trend with temperature for single component materials (e.g. [16]), while it shows the above mentioned nonlinear alternation of increasing and decreasing stages with increasing temperature, as well as some hysteresis upon temperature cycling, for composite (i.e., multi-component) materials (e.g. [17]). Similar trends have been experimentally observed also in the thermal evolution of material strength properties, such as the uniaxial tensile or compressive strength (e.g., [10], [24]).

Due to the above mentioned heterogeneity of thermo-mechanical properties among different refractory materials, it is not possible to formulate a general constitutive model that can simulate properly all typical aspects of material response. Any thermo-mechanical constitutive model must be formulated and calibrated for the given composition and forming process that are peculiar of the specific material at hand, resulting in a somewhat restricted range of applicability.

In this work, some typical aspects of material response are considered, that apply to broad families of rock-like materials. Despite the main focus of this paper being on refractory materials, for which there is a certain availability of experimental measurements at high temperature, this work aims at proposing a general framework that could be easily applicable in an interdisciplinary context, to investigate the behaviour of geomaterials subjected to very high temperature. For example the same modelling approach can be applied to the natural conunterparts of refractories, namely rocks, that in some contexts can be subjected to analogous thermo-mechanical loading conditions, such as those acting in shallow crustal phenomena (shear heating of faults and of rockslide slip planes, [29], [2]).

The limitations and implications of both basic and more complex constitutive assumptions concerning the thermo-elastic material response are discussed. In particular, the cases of reversible and irreversible thermo-mechanical behaviour upon thermal cycling at low applied stresses (i.e. within the elastic domain) are addressed. To capture irreversible response, a thermo-elastic model is also developed in the framework of elasto-plastic coupling (i.e., coupling between thermal, elastic and plastic properties is considered). A discussion follows on how the two thermo-mechanical elastic (reversible and irreversible) frameworks can be combined with a plastic driver to obtain a thermo-dynamically consistent, general elasto-plastic framework for refractory materials. Finally, the proposed framework is validated by reproducing numerically some experimentally observed trends. 


\section{Notation:}

The following tensorial product will be used:

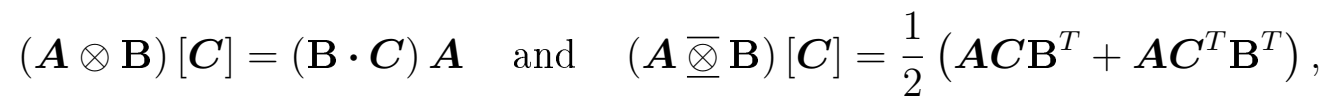

for every second-order tensor $\boldsymbol{A}, \mathrm{B}$ and $\boldsymbol{C}$.

\section{Basic assumptions:}

The usual strain decomposition into the elastic and plastic strains will be assumed below, namely

$$
\boldsymbol{\epsilon}=\boldsymbol{\epsilon}_{e}+\boldsymbol{\epsilon}_{p} \quad \text { and } \quad \dot{\boldsymbol{\epsilon}}=\dot{\boldsymbol{\epsilon}}_{e}+\dot{\boldsymbol{\epsilon}}_{p}
$$

where the dot stands for time derivative.

Moreover, following [27], the same decomposition is assumed to hold true for the entropy (per unit initial volume), namely

$$
\eta=\eta_{e}+\eta_{p} \quad \text { and } \quad \dot{\eta}=\dot{\eta}_{e}+\dot{\eta}_{p}
$$

Thus, absolute temperature $\theta$ plays the role of a stress-like quantity (cf. [27]), whereas the entropy plays the role of the corresponding (conjugated) strain-like quantity.

\section{Thermodynamic potentials with reversible ther- mal behaviour}

Let us first consider a refractory material undergoing negligible irreversible behaviour when subjected to a thermal loading-unloading cycle, under a small applied stress. The thermodynamic potential proposed in this Section assumes a perfectly reversible behaviour, thus the thermo-mechanical properties are assumed to remain unchanged after either a thermal, or mechanical, loading-unloading cycle, as long as the stress state remains within the yield surface.

Under non-isothermal conditions, the free energy per unit undeformed volume is expressed as

$$
\psi=\psi\left(\boldsymbol{\epsilon}_{e}, \theta\right)
$$

where $\boldsymbol{\epsilon}_{e}$ is the small strain elasticity tensor and $\theta$ is the absolute temperature. Upon time differentiation, we obtain

$$
\dot{\psi}=\frac{\partial \psi}{\partial \boldsymbol{\epsilon}_{e}} \cdot \dot{\boldsymbol{\epsilon}}_{e}+\frac{\partial \psi}{\partial \theta} \dot{\theta}
$$


The definition of internal dissipation $\mathcal{D}_{\text {int }}$ involves the work done by the total stress with the corresponding work-conjugate strain rate, thus

$$
\mathcal{D}_{\text {int }}=\theta \dot{\eta}+\boldsymbol{\sigma} \cdot \dot{\boldsymbol{\epsilon}}-\dot{e}
$$

where $\boldsymbol{\sigma}$ is the stress tensor and $e$ is the internal energy function $e=e\left(\boldsymbol{\epsilon}_{e}, \eta_{e}\right)$, which is related to the free energy density through a Legendre transformation, as follows

$$
\psi\left(\boldsymbol{\epsilon}_{e}, \theta\right)=e\left(\boldsymbol{\epsilon}_{e}, \eta_{e}\right)-\eta_{e} \theta
$$

In the case of purely thermoelastic response $\left(\dot{\boldsymbol{\epsilon}}=\dot{\boldsymbol{\epsilon}}_{e}\right.$ and $\left.\dot{\eta}=\dot{\eta}_{e}\right)$, the insertion of eqn. (6) into eqn. (5) leads to

$$
\mathcal{D}_{i n t}=\boldsymbol{\sigma} \cdot \dot{\boldsymbol{\epsilon}}_{e}-\eta_{e} \dot{\theta}-\dot{\psi}
$$

and the internal dissipation must vanish, $\mathcal{D}_{\text {int }}=0$, thus, through standard arguments [3], the following constitutive assumptions are obtained

$$
\boldsymbol{\sigma}=\frac{\partial \psi}{\partial \boldsymbol{\epsilon}_{e}}, \eta_{e}=-\frac{\partial \psi}{\partial \theta}
$$

It should be noted that the above outlined approach, at variance with the thermoelastic theory proposed by Green and Naghdi [13] and further developed by [20], follows classic thermoelasticity, thus involving energy dissipation due to heat transfer. In the following, we analyse the implications of adopting the simplest possible class of $\psi$ that can be deduced from published experimental results on refractory materials, namely

$$
\begin{gathered}
\psi\left(\boldsymbol{\epsilon}_{e}, \theta\right)=\frac{1}{2} K(\theta)\left(\operatorname{Tr}\left(\boldsymbol{\epsilon}_{e}\right)-A(\theta)\left(\theta-\theta_{0}\right)\right)^{2}+G(\theta) \mathbf{e}_{e} \cdot \mathbf{e}_{e}+ \\
-C(\theta)\left[\theta \log \left(\frac{\theta}{\theta_{0}}\right)-\left(\theta-\theta_{0}\right)\right]
\end{gathered}
$$

where $\mathbf{e}_{e}$ is the deviatoric elastic strain tensor, $K(\theta), G(\theta), A(\theta)$ and $C(\theta)$ are suitable interpolation functions of the absolute temperature $\theta$, evaluated from the available experimental data, and $\theta_{0}$ is the initial temperature. An isotropic response is assumed in eqn.(9) and functions $K(\theta)$ and $G(\theta)$ represent the temperature dependent bulk and shear moduli. The functions $A(\theta)$ and $C(\theta)$ are related to the temperature dependent heat volumetric expansion coefficient and to the constant-volume heat capacity, respectively. The choice of the most suitable interpolation functions is discussed hereafter.

From eqns. (8) and (9) we can deduce that

$$
\boldsymbol{\sigma}=\frac{\partial \psi}{\partial \boldsymbol{\epsilon}_{e}}=K(\theta)\left(\operatorname{Tr}\left(\boldsymbol{\epsilon}_{e}\right)-A(\theta)\left(\theta-\theta_{0}\right)\right) \boldsymbol{I}+2 G(\theta) \mathbf{e}_{e}
$$


where $\boldsymbol{I}$ the second order identity tensor, thus the resulting elastic tangent stiffness matrix depends only on temperature, through

$$
\mathbb{D}=\frac{\partial^{2} \psi}{\partial \boldsymbol{\epsilon}_{e}^{2}}=\left(K(\theta)+\frac{2}{3} G(\theta)\right) \boldsymbol{I} \otimes \boldsymbol{I}+2 G(\theta) \underline{\boldsymbol{I}} \underline{\bar{\nabla}} .
$$

Since non-isothermal experiments are usually performed at low applied stress (i.e. at atmospheric pressure), the complementary potential of the free energy density function, the so-called free enthalpy, must be found through a partial Legendre transformation

$$
\varphi(\boldsymbol{\sigma}, \theta)=\psi\left(\boldsymbol{\epsilon}_{e}(\boldsymbol{\sigma}), \theta\right)-\boldsymbol{\sigma} \cdot \boldsymbol{\epsilon}_{e}
$$

which is equal to

$$
\begin{aligned}
\varphi(\boldsymbol{\sigma}, \theta)=-\frac{\operatorname{Tr}(\boldsymbol{\sigma})}{K(\theta)} & \left(\frac{\operatorname{Tr}(\boldsymbol{\sigma})}{2}+A(\theta)\left(\theta-\theta_{0}\right) K(\theta)\right)-\frac{1}{4 G(\theta)} \boldsymbol{\sigma}_{D} \cdot \boldsymbol{\sigma}_{D}+ \\
& -C(\theta)\left[\theta \log \left(\frac{\theta}{\theta_{0}}\right)-\left(\theta-\theta_{0}\right)\right]
\end{aligned}
$$

where $\boldsymbol{\sigma}_{D}$ is the deviatoric component of $\boldsymbol{\sigma}$. The strain tensor can be immediately deduced as

$$
\boldsymbol{\epsilon}_{e}=-\frac{\partial \varphi}{\partial \boldsymbol{\sigma}}=\left(\frac{\operatorname{Tr}(\boldsymbol{\sigma})}{K(\theta)}+A(\theta)\left(\theta-\theta_{0}\right)\right) \boldsymbol{I}+\frac{1}{2 G(\theta)} \boldsymbol{\sigma}_{D}
$$

and its derivative with respect to temperature, representing the thermal expansion coefficient at constant stress, is obtained as

$$
\boldsymbol{\alpha}_{\boldsymbol{\sigma}}=\frac{\partial \boldsymbol{\epsilon}}{\partial \theta}=-\frac{\operatorname{Tr}(\boldsymbol{\sigma})}{(K(\theta))^{2}} K^{\prime}(\theta) \boldsymbol{I}+\left(A^{\prime}(\theta)\left(\theta-\theta_{0}\right)+A(\theta)\right) \boldsymbol{I}-\frac{G^{\prime}(\theta)}{2(G(\theta))^{2}} \boldsymbol{\sigma}_{D} .
$$

Equation (15) implies that the thermal expansion coefficient is a tensor quantity and depends on the applied stress. In usual applications, due to technical difficulties, the thermal expansion coefficient is evaluated at atmospheric pressure, which is usually negligible compared to the magnitude of elastic moduli, thus the thermal expansion coefficient can be practically rewritten as independent of the applied stress and isotropic

$$
\boldsymbol{\alpha}_{\boldsymbol{\sigma}} \approx\left(A^{\prime}(\theta)\left(\theta-\theta_{0}\right)+A(\theta)\right) \boldsymbol{I} .
$$

As a result, under negligible applied stress, the thermal expansion coefficient takes the usual meaning (i.e., it is equal in all directions) and depends only on temperature.

The entropy at constant stress is given by

$$
\eta_{e}^{\sigma}=-\frac{\partial \varphi}{\partial \theta}=-\frac{(\operatorname{Tr}(\boldsymbol{\sigma}))^{2}}{(K(\theta))^{2}} K^{\prime}(\theta)+\operatorname{Tr}(\boldsymbol{\sigma})\left(A^{\prime}(\theta)\left(\theta-\theta_{0}\right)+A(\theta)\right)
$$




$$
-\frac{1}{4(G(\theta))^{2}} G^{\prime}(\theta) \boldsymbol{\sigma}_{D} \cdot \boldsymbol{\sigma}_{D}-C^{\prime}(\theta)\left[\theta \log \left(\frac{\theta}{\theta_{0}}\right)-\left(\theta-\theta_{0}\right)\right]-C(\theta) \log \left(\frac{\theta}{\theta_{0}}\right),
$$

thus the specific heat capacity at constant pressure writes

$$
\begin{gathered}
c_{p}=-\theta \frac{\partial \eta_{e}^{\sigma}}{\partial \theta}=-\theta \frac{(\operatorname{Tr}(\boldsymbol{\sigma}))^{2}}{(K(\theta))^{2}}\left(2 \frac{\left(K^{\prime}(\theta)\right)^{2}}{K(\theta)}-K^{\prime \prime}(\theta)\right)+ \\
-\theta \operatorname{Tr}(\boldsymbol{\sigma})\left(A^{\prime \prime}(\theta)\left(\theta-\theta_{0}\right)+2 A^{\prime}(\theta)\right)-\frac{\theta_{0}}{4(G(\theta))^{2}}\left(2 \frac{\left(G^{\prime}(\theta)\right)^{2}}{G(\theta)}-G^{\prime \prime}(\theta)\right) \boldsymbol{\sigma}_{D} \cdot \boldsymbol{\sigma}_{D}+ \\
+C^{\prime \prime}(\theta) \theta\left[\theta \log \left(\frac{\theta}{\theta_{0}}\right)-\left(\theta-\theta_{0}\right)\right]+2 C^{\prime}(\theta) \theta \log \left(\frac{\theta}{\theta_{0}}\right)+C(\theta),
\end{gathered}
$$

which also depends on the applied stress. The experimental evaluation of $c_{p}$ is typically performed at negligible applied stress, thus $c_{p}$ becomes

$$
c_{p} \approx C^{\prime \prime}(\theta) \theta\left[\theta \log \left(\frac{\theta}{\theta_{0}}\right)-\left(\theta-\theta_{0}\right)\right]+2 C^{\prime}(\theta) \theta \log \left(\frac{\theta}{\theta_{0}}\right)+C(\theta) .
$$

and practically depends only on temperature.

In the solution of a typical boundary value problem, the entropy at constant strain $\eta_{e}$ is usually employed and can be derived from the free energy (eqn. (9)) as

$$
\begin{gathered}
\eta_{e}=-\frac{\partial \psi}{\partial \theta}=-\frac{1}{2} K^{\prime}(\theta)\left(\operatorname{Tr}\left(\boldsymbol{\epsilon}_{e}\right)-A(\theta)\left(\theta-\theta_{0}\right)\right)^{2}+ \\
+K(\theta)\left(\operatorname{Tr}(\boldsymbol{\epsilon})-A(\theta)\left(\theta-\theta_{0}\right)\right)\left(A^{\prime}(\theta)\left(\theta-\theta_{0}\right)+A(\theta)\right)+ \\
-G^{\prime}(\theta) \mathbf{e}_{e} \cdot \mathbf{e}_{e}-C^{\prime}(\theta)\left[\theta \log \left(\frac{\theta}{\theta_{0}}\right)-\left(\theta-\theta_{0}\right)\right]-C(\theta) \log \left(\frac{\theta}{\theta_{0}}\right) .
\end{gathered}
$$

Experimental tests typically supply data concerning the thermal dependence of elastic stiffness, the variation of thermal expansion coefficient and of specific heat capacity with temperature, both evaluated at negligible applied stress. It can be observed that all quantities expressed by eqns. (11), (16) and (19) are decoupled from each other. Thus, it is possible to accurately interpolate each experimental plot (obtained at negligible applied stress) by using suitable interpolation functions $K(\theta), G(\theta), A(\theta)$ and $C(\theta)$, which can be chosen as complex as needed. This feature constitutes a major advantage of this simple approach.

Thus, for instance, the interpolating functions useful to simulate experimental results about thermal dependency of stiffness in sintered alumina (e.g., see Fig. 2 of [32], or Fig. 4 of [16]), shown in Figure 1a, can be set as follows

$$
\begin{gathered}
K(\theta)=a_{K}-b_{K}\left(\theta-\theta_{0}\right)-c_{K} \operatorname{Tanh}\left(\frac{\theta-\theta_{K}}{d_{K}}\right) \\
G(\theta)=K(\theta) \frac{2(1+\nu)}{3(1-2 \nu)}
\end{gathered}
$$


where $\nu$ is the constant Poisson's ratio and

$$
\begin{aligned}
& A(\theta)=a_{A}+b_{A}\left(\frac{\theta-\theta_{A}}{\theta_{0}}\right)^{e_{A}} \\
& C(\theta)=a_{C}+b_{C}\left(\frac{\theta-\theta_{A}}{\theta_{0}}\right)^{e_{C}}
\end{aligned}
$$

where $\theta_{i}$ and $a_{i}, b_{i}$ and $d_{i}$ (with $i=K, A, C$ ) are coefficients that must be calibrated on the experimental results. The parameter values chosen to reproduce the thermo-mechanical behaviour of sintered alumina are reported in Table 1 (Set 1). Relevant initial conditions are reported in Table 2 (Set 1). It is worth remarking that there is no restriction on the choice of the most suitable interpolation functions.

The simplicity of this approach has, however, important implications that should be remarked. First of all, let us recall that this approach does not permit to simulate any sort of irreversibility induced by a thermal loading-unloading cycle, as long as the stress lies inside the yield surface. Although such reversible behaviour does not apply to all refractory materials, the simplicity of this approach can be appealing in many applications involving refractory materials with simple thermal response (i.e. with negligible hysteresis) or even materials exhibiting more complex thermal behaviour, when subjected only to monotonic thermal loading conditions.

It should also be remarked that the proposed model is calibrated along simple loading conditions, i.e. the thermal properties are evaluated at negligible applied stress and varying temperature, whereas the elastic stiffness is measured at different temperatures, starting from initially negligible (atmospheric) pressure. This implies that the thermal potentials described above may produce unexpected responses, when applied to more general thermal and mechanical loading conditions. Figure 1 shows the simulated temperature dependence of elastic stiffness, specific heat capacity and longitudinal deformation (whose slope represents the thermal expansion coefficient), for a sample subjected to thermal loading under three different, constant applied stress levels. It can be observed that, as long as the applied load is smaller than about $100 \mathrm{MPa}$ (corresponding to about $5 \%$ of the elasticity modulus $E$ at room temperature), the thermo-mechanical coupling is small, i.e. the difference between the plots obtained at 0.1 and at $100 \mathrm{MPa}$ of applied stress is negligible. As a result, the experimental behaviour exhibited at atmospheric pressure is expected to be meaningful also at slightly higher applied stresses. In contrast, the thermo-mechanical coupling may unexpectedly change when the applied stress is large compared to the elastic stiffness. This is evident in Figures 1b and 1c, showing that if the sample is subject to a $1000 \mathrm{MPa}$ stress level, both the thermal evolution of specific heat capacity and of thermal expansion coefficient significantly change their trend in the higher temperature range. 
Further, it can be deduced from Figure 1a that the interpolating function (eqn. 21) implies a non-negligible decrease of stiffness (for instance due to a melting phase) at high temperatures. Such decrease of stiffness can reflect, for example, the influence of a glassy matrix, and is responsible for the unexpected change in the thermally induced longitudinal deformation and specific heat under large applied stress (Figures 1b and 1c). In other words, the thermal and mechanical material responses become more deeply coupled when the sample is loaded by a large applied stress, even though the proposed interpolation functions (eqns. 21-24) are not, apparently, mechanically coupled.

The thermodynamic potential proposed in eqn. (9) assumes a perfect reversible behaviour, thus plastic strains and any irreversible fraction of entropy variation must be defined by the plastic flow rule. In the case of irreversible behaviour $\left(\dot{\boldsymbol{\epsilon}}_{p} \neq \mathbf{0}\right.$ and $\dot{\eta}_{p} \neq 0$ ), the internal dissipation (eqn. 5) is not null and becomes

$$
\mathcal{D}_{i n t}=\boldsymbol{\sigma} \cdot \dot{\boldsymbol{\epsilon}}_{p}+\theta \dot{\eta}_{p}
$$

As a result, if the yield function is assumed to take the following form

$$
\mathcal{F}(\boldsymbol{\sigma}, \theta, \kappa)=0
$$

where $\kappa$ is a set of internal variables, then, to achieve maximum dissipation, the flow rule must be associated [27]. Thus, the increments of plastic strain-like quantities (i.e. $\boldsymbol{\epsilon}_{p}$ and $\eta_{p}$ ) must be orthogonal to the yield surface $\mathcal{F}$, namely

$$
\dot{\boldsymbol{\epsilon}}_{p}=\dot{\Lambda} \frac{\partial \mathcal{F}(\boldsymbol{\sigma}, \theta, \kappa)}{\partial \boldsymbol{\sigma}} \quad \text { and } \quad \dot{\eta}_{p}=\dot{\Lambda} \frac{\partial \mathcal{F}(\boldsymbol{\sigma}, \theta, \kappa)}{\partial \theta} .
$$

where $\dot{\Lambda}$ is the plastic multiplier.

\section{Thermodynamic potentials with irreversible ther- mal behaviour}

The thermodynamic potentials discussed in Section 2 were based on the assumption of a perfectly reversible behaviour under a thermal loading/unloading cycle, performed at negligible applied stress. In other terms, a thermal cycle performed at negligible applied stresses is assumed to leave unchanged the thermal and mechanical properties of the refractory material. Unfortunately, this assumption does not always hold (especially in composite materials) and in most practical cases, an irreversible response is observed. This is due to many reasons, such as phase change, chemical reactions and melting of some constituents with the consequent curing of microcracks. To describe these effects, the thermodynamic potentials must account for meaningful and physically-based internal variables, that are capable of describing the relevant micro-scale changes, such as the relative amount of the different phases (if a phase change occurs), the extent of chemical reactions or the amount of internal defects. 


\begin{tabular}{|c|c|c|c|c|c|}
\hline \multicolumn{3}{|r|}{ Alumina } & \multicolumn{3}{|c|}{ Composite refractories } \\
\hline Param. & Units & Set 1 & Set 2 & Set 3 & Set 4 \\
\hline$a_{K}$ & $\mathrm{~Pa}$ & $0.73 \times 10^{10}$ & $-0.71 \times 10^{10}$ & $-0.71 \times 10^{10}$ & $-2.3 \times 10^{8}$ \\
\hline$b_{K}$ & $\mathrm{~Pa} / \mathrm{K}$ & $1.0 \times 10^{6}$ & $8.33 \times 10^{6}$ & $8.33 \times 10^{6}$ & $2.78 \times 10^{5}$ \\
\hline$c_{K}$ & $\mathrm{~Pa}$ & $0.18 \times 10^{10}$ & $9.03 \times 10^{10}$ & $9.03 \times 10^{10}$ & $3 \times 10^{9}$ \\
\hline$d_{K}$ & $\mathrm{~K}$ & 150 & 160 & 160 & 160 \\
\hline$\theta_{K}$ & $\mathrm{~K}$ & 1900 & 1550 & 1550 & 1550 \\
\hline$a_{A}$ & $\mathrm{~K}^{-1}$ & $2 \times 10^{-} 6$ & $0.5 \times 10^{-6}$ & $0.5 \times 10^{-6}$ & $0.5 \times 10^{-6}$ \\
\hline$b_{A}$ & $\mathrm{~K}^{-1}$ & $5.48 \times 10^{-} 6$ & $1.37 \times 10^{-6}$ & $1.37 \times 10^{-6}$ & $1.37 \times 10^{-6}$ \\
\hline$e_{A}$ & - & 0.3 & 0.3 & 0.3 & 0.3 \\
\hline$\theta_{A}$ & $\mathrm{~K}$ & 250 & 250 & 250 & 250 \\
\hline$a_{C}$ & $\mathrm{~K}^{-1}$ & $2.6 \times 10^{6}$ & $2.6 \times 10^{6}$ & $2.6 \times 10^{6}$ & $2.6 \times 10^{6}$ \\
\hline$b_{C}$ & $\mathrm{~K}^{-1}$ & $8.77 \times 10^{5}$ & $8.77 \times 10^{5}$ & $8.77 \times 10^{5}$ & $8.77 \times 10^{5}$ \\
\hline$e_{C}$ & - & 0.3 & 0.3 & 0.3 & 0.3 \\
\hline$\nu$ & - & 0.1 & 0.1 & 0.1 & 0.1 \\
\hline$r_{K}$ & $\mathrm{~Pa}$ & - & $1.88 \times 10^{10}$ & $4.23 \times 10^{10}$ & $6.26 \times 10^{8}$ \\
\hline$e_{K}$ & $\mathrm{~Pa}$ & - & $3.75 \times 10^{10}$ & $8.44 \times 10^{10}$ & $1.25 \times 10^{9}$ \\
\hline$k_{1}$ & $\mathrm{~K}^{-2}$ & - & $2.25 \times 10^{10}$ & $1.13 \times 10^{10}$ & $2.25 \times 10^{10}$ \\
\hline$k_{2}$ & $\mathrm{~K}^{-2}$ & - & $0.2 \times 10^{10}$ & $0.25 \times 10^{10}$ & $0.2 \times 10^{10}$ \\
\hline$k_{3}$ & - & - & $4 \times 10^{3}$ & $4 \times 10^{3}$ & $9.6 \times 10^{3}$ \\
\hline$A$ & - & - & 0.8 & 0.8 & 0.8 \\
\hline$\theta_{F}$ & K & - & 973 & 374 & 973 \\
\hline$\theta_{R}$ & $\mathrm{~K}$ & - & 673 & 200 & 673 \\
\hline$\theta_{C}$ & $\mathrm{~K}$ & - & 1173 & 1173 & 1173 \\
\hline$d_{C}$ & $\mathrm{~K}$ & - & 300 & 300 & 200 \\
\hline$k_{\epsilon}$ & - & - & $-7.2 \times 10^{-3}$ & $-3.6 \times 10^{-3}$ & $-7.2 \times 10^{-3}$ \\
\hline$a_{R C}$ & $\mathrm{~Pa}$ & - & $10 \times 10^{6}$ & $5.8 \times 10^{7}$ & $2.8 \times 10^{7}$ \\
\hline$b_{R C}$ & $\mathrm{~Pa}$ & - & $8 \times 10^{6}$ & $5.6 \times 10^{7}$ & $2.5 \times 10^{7}$ \\
\hline$\theta_{M}$ & $\mathrm{~K}$ & - & 1400 & 1400 & 1400 \\
\hline$r_{R C}$ & $\mathrm{~K}$ & - & 200 & 200 & 200 \\
\hline$\lambda$ & - & - & $0.2-0.03$ & $0.2-0.03$ & $0.2-0.03$ \\
\hline
\end{tabular}

Table 1: Summary of the main model parameters adopted for the different materials.

\begin{tabular}{llllll}
\hline Param. & Units & Set 1 & Set 2 & Set 3 & Set 4 \\
\hline$P_{c}-N_{c c} \sigma_{r c}$ & $\mathrm{~Pa}$ & - & $5 \times 10^{6}$ & $10 \times 10^{6}$ & $10 \times 10^{6}$ \\
$N_{c f}$ & - & - & 0.6 & 0.43 & 0.6 \\
$\sigma_{0}$ & $\mathrm{~Pa}$ & $10^{5}$ & $10^{5}$ & $10^{5}$ & $10^{5}$ \\
$\theta_{0}$ & $\mathrm{~K}$ & 290 & 290 & 290 & 290 \\
\hline
\end{tabular}

Table 2: Initial values of key parameters adopted in the simulations. 


\subsection{Choice of internal variables}

To formulate suitable thermodynamic potentials, account was taken for the experimental evidence available in the literature on composite refractories (e.g. [31], [16], [17] and [32]), where the existence of three micro-scale mechanisms can be schematically outlined in the evolution of the (dynamically measured) Young's modulus $E$ versus temperature. Such mechanisms, emerging during thermal loading starting from ambient temperature up to about $1500{ }^{\circ} \mathrm{C}$, consist of 1) an initial, slight decrease of $E$ due to reducing atomic bonding stiffness [17] (and/or dehydration if the material at hand is a castable concrete, see [16]);2) a subsequent increase of $E$ due to closing of microcracks (induced from differential thermal expansion of the various constituents) and 3) a final reduction of $E$ due to (partial) melting of some constituents.

Based on the above experimental observations, the fraction of closed microcracks, $N_{c c}$ (generally due to both differential thermal expansion and mechanically induced volumetric strain), and the fraction of cured microcracks $N_{c f}$ (due to the possible melting of some phases) can be chosen as internal variables to feature in the thermodynamic potential accounting for irreversible behaviour. In particular $N_{c f}$ can vary between 0 and $N_{c c}$, because the cured microcracks are a subset of the closed microcracks. As a result, the number of closed microcracks $N_{c c}$ ranges between $N_{c f}$ and a limiting value $N_{\text {lim }}=1$ (Figure 2). The condition $N_{c f}=0$ represents a limiting situation of completely decemented material (exhibiting only a frictional resistance), whereas the condition $N_{c c}=N_{c f}$ is representative of a situation in which all microcracks that are not cemented are open, typically occurring at low temperature (and low applied stress). In contrast, the case $N_{c c}=N_{c f}=N_{\text {lim }}$ represents the limiting situation when the material is completely cemented and no microscopic defect is present. Thus, subject to the above constraints, both $N_{c c}$ and $N_{c f}$ can range between 0 and 1 .

It is now required to define the evolution laws of the newly introduced internal variables, $N_{c c}$ and $N_{c f}$. $N_{c c}$ is generally expected to depend on on temperature (the different thermal expansion of the various constituents is expected to affect the pore volume, thus also affecting $N_{c c}$ ) and on the the applied strain (in fact, a compressive volume strain is expected to decrease the pore volume and consequently increase $N_{c c}$ ). Moreover, $N_{c c}$ should be rigorously represented by a tensor quantity, to account for the fact that the normal directions to closed microcracks might generally have a non-isotropic distribution. Both anisotropy and strain dependency have been neglected in the formulation presented below for the sake of simplicity, and due to a lack of experimental evidence. In particular, neglecting the dependence on the applied strain is acceptable if the materials at hand exhibit a high stiffness and a non-negligible thermal expansion, and are subject to very large temperature variations, which is typically the case of refractory materials. On the other hand, neglecting the dependence of $N_{c c}$ on the applied strain is not acceptable in other applications, such as the analysis of isostatic pressing of a 
ceramic powder.

The following empirical law is proposed for the evolution of $N_{c c}$

$$
N_{c c}=\frac{N_{l i m}+N_{c f}}{2}+\frac{N_{l i m}-N_{c f}}{2} \operatorname{Tanh}\left(\frac{\theta-\theta_{C}}{d_{C}}\right),
$$

where $\theta_{C}$ is the temperature corresponding to the highest rate of microcracks closing, and $d_{C}$ is a constitutive parameter. Note that $N_{c f}$ and $N_{l i m}$ are the limiting values of $N_{c c}$, and that the proposed law implies full reversibility if $N_{c f}$ is constant.

It is worth anticipating here that the closure of microcracks is assumed to lead to an increase of elastic stiffness. As a result, introducing a dependency of $N_{c c}$ on strain could permit a reliable simulation of the observed increase of elastic properties induced by static compaction.

The evolution law of $N_{c f}$ with temperature cannot be established along the lines of the evolution of $N_{c c}$ (eqn. 28), because the effects of temperature on $N_{c f}$ are assumed irreversible. Moreover, $N_{c f}$ is assumed to also depend on plastic strain, which can induce new microcracks. Hence, the following relationship is proposed

$$
\begin{aligned}
\dot{N}_{c f}=k_{1}\left(N_{c c}-\right. & \left.N_{c f}\right)<\frac{\theta-\theta_{F}}{\theta_{0}}><\dot{\theta}>-k_{2} N_{c f}<\frac{\theta_{R}-\theta}{\theta_{0}}><-\dot{\theta}>+ \\
& -k_{3} N_{c f} \sqrt{(1-A) \operatorname{Tr}\left(\dot{\boldsymbol{\epsilon}}_{p}\right)^{2}+A \operatorname{Tr}\left(\dot{\mathbf{e}}_{p} \cdot \dot{\mathbf{e}}_{p}\right)},
\end{aligned}
$$

where $A$ and $k_{3}$ are constitutive parameters (with $0 \leq A \leq 1$ ) governing the increase of microcracks due to plastic strains $\boldsymbol{\epsilon}_{p}\left(\mathbf{e}_{p}\right.$ is the deviatoric component of the plastic strain), $\theta_{F}$ and $\theta_{R}$ are the temperature values at which curing (due to melting) and rupture of microcracks, respectively, mostly occurs. $k_{1}$ and $k_{2}$ are constitutive coefficients (having units of $1 / \mathrm{K}$ ) and $<>$ denotes the Macaulay brackets, thus the first term applies for $\theta>\theta_{F}$ and $\dot{\theta}>0$, whereas the second term applies for $\theta<\theta_{R}$ and $\dot{\theta}<0$. As a result, the first term of eqn. (29) rules microcracks curing (occurring at $\theta>\theta_{F}$ and $\dot{\theta}>0$ ), whereas the second term rules microcracks rupture due to the decrease of temperature (for $\theta<\theta_{R}$ and $\dot{\theta}<0$ ). Finally, the last term of eqn. (29) describes the formation of new microcracks due to plastic loading. Note that $N_{c c}$ and 0 are the limiting values of $N_{c f}$.

\subsection{Formulation of thermodynamic potentials}

The proposed free energy density function has the following functional dependency

$$
\psi=\widetilde{\psi}\left(\boldsymbol{\epsilon}_{e}, \theta, N_{c c}, N_{c f}\right)
$$


where $N_{c c}$ and $N_{c f}$ could be considered to play the role of internal variables depending on the previous thermal and mechanical loading histories. However, since both $N_{c c}$ and $N_{c f}$ depend on temperature and on plastic strains through eqns. (28) and (29), the functional dependency of $\psi$ can equivalently, but more consistently, be expressed as

$$
\psi=\psi\left(\boldsymbol{\epsilon}_{e}, \theta, \boldsymbol{\epsilon}_{p}\right) .
$$

In any case, due to the above assumptions, a thermal loading-unloading cycle performed at negligible applied stress affects the final properties of the material, due to the irreversible changes of $N_{c c}$ and $N_{c f}$ induced by temperature variations through eqns. (28) and (29).

The free energy density function is assumed to take the form

$$
\begin{gathered}
\psi\left(\boldsymbol{\epsilon}_{e}, \theta, \boldsymbol{\epsilon}_{p}\right)=\frac{1}{2} K\left(\theta, \boldsymbol{\epsilon}_{p}\right)\left(\operatorname{Tr}\left(\boldsymbol{\epsilon}_{e}\right)-A(\theta)\left(\theta-\theta_{0}\right)+k_{\epsilon} N_{c f}\right)^{2}+ \\
+G\left(\theta, \boldsymbol{\epsilon}_{p}\right) \mathbf{e}_{e} \cdot \mathbf{e}_{e}+C(\theta)\left[\theta \log \left(\frac{\theta}{\theta_{0}}\right)-\left(\theta-\theta_{0}\right)\right]
\end{gathered}
$$

where $k_{\epsilon}$ is a constitutive parameter describing the volume deformation due to the curing of microcracks and functions $K\left(\theta, \boldsymbol{\epsilon}_{p}\right)$ and $G\left(\theta, \boldsymbol{\epsilon}_{p}\right)$ represent the bulk and shear moduli. These are expressed by the following extension of eqn. (21)

$$
K\left(\theta, \boldsymbol{\epsilon}_{p}\right)=a_{K}-b_{K}\left(\theta-\theta_{0}\right)-c_{K} \operatorname{Tanh}\left(\frac{\theta-\theta_{K}}{d_{K}}\right)+r_{K} N_{c c}+e_{K} N_{c f}
$$

where $a_{K}, b_{K}, c_{K}, d_{K}, e_{K}, r_{K}$ and $\theta_{K}$ are constitutive parameters, and

$$
G\left(\theta, \boldsymbol{\epsilon}_{p}\right)=K\left(\theta, \boldsymbol{\epsilon}_{p}\right) \frac{2(1+\nu)}{3(1-2 \nu)}
$$

with a constant Poisson's ratio $\nu$.

It is worth observing that the elastic stiffness depends directly on temperature (thus, accounting for the experimentally observed reduction of stiffness due to melting of some constituents, at high temperature), on $N_{c c}$ (describing the increase of stiffness due to closing of microcracks) and on $N_{c f}$ (describing the increase of stiffness due to the curing of microcracks). Thus the irreversibility of elastic stiffness variation versus temperature is introduced in eqn. (33) through the dependence on $N_{c c}$ and $N_{c f}$. The interpolating functions $A(\theta)$ and $C(\theta)$ are assumed the same as in eqns. (23-24).

The free enthalpy can be easily deduced along the lines of eqn. (12), as

$$
\varphi\left(\boldsymbol{\sigma}, \theta, \boldsymbol{\epsilon}_{p}\right)=-\operatorname{Tr}(\boldsymbol{\sigma})\left(\frac{\operatorname{Tr}(\boldsymbol{\sigma})}{2 K\left(\theta, \boldsymbol{\epsilon}_{p}\right)}+A(\theta)\left(\theta-\theta_{0}\right)-k_{\epsilon} N_{c f}\right)+
$$




$$
-\frac{1}{4 G\left(\theta, \boldsymbol{\epsilon}_{p}\right)} \boldsymbol{\sigma}_{D} \cdot \boldsymbol{\sigma}_{D}+C(\theta)\left[\theta \log \left(\frac{\theta}{\theta_{0}}\right)-\left(\theta-\theta_{0}\right)\right],
$$

from which the elastic strain tensor is derived as

$$
\boldsymbol{\epsilon}_{e}=\left(\frac{\operatorname{Tr}(\boldsymbol{\sigma})}{K\left(\theta, \boldsymbol{\epsilon}_{p}\right)}+A(\theta)\left(\theta-\theta_{0}\right)-k_{\epsilon} N_{c f}\right) \boldsymbol{I}+\frac{1}{2 G\left(\theta, \boldsymbol{\epsilon}_{p}\right)} \boldsymbol{\sigma}_{D}
$$

whereas from eqn. (32), the stress tensor is obtained as

$$
\begin{aligned}
& \boldsymbol{\sigma}=-\frac{\partial \psi}{\partial \boldsymbol{\epsilon}_{e}}=K\left(\theta, \boldsymbol{\epsilon}_{p}\right)\left(\operatorname{Tr}\left(\boldsymbol{\epsilon}_{e}\right)-A(\theta)\left(\theta-\theta_{0}\right)+k_{\epsilon} N_{c f}\right) \boldsymbol{I}+ \\
&+2 G\left(\theta, \boldsymbol{\epsilon}_{p}\right) \mathbf{e}_{e} .
\end{aligned}
$$

It is worth remarking that the dependence of elastic properties and elastic strain on $N_{c f}$ (eqns. 33, 34 and 36) implies a dependency on plastic strains, in the form of damage, i.e. a progressive degradation of elastic stiffness induced by plastic strains. This sort of effect is denoted in the literature as elasto-plastic coupling, and was first postulated by Hueckel and Maier [15].

The entropy per unit initial volume is obtained by deriving the potential $\psi$ with respect to temperature (see eqns. 17 and 20), namely

$$
\begin{gathered}
\eta_{e}=-\frac{\partial \psi}{\partial \theta}=-\frac{1}{2} \frac{\partial K\left(\theta, \boldsymbol{\epsilon}_{p}\right)}{\partial \theta}\left(\operatorname{Tr}\left(\boldsymbol{\epsilon}_{e}\right)-A(\theta)\left(\theta-\theta_{0}\right)+k_{\epsilon} N_{c f}\right)^{2}+ \\
+K\left(\theta, \boldsymbol{\epsilon}_{p}\right)\left(\operatorname{Tr}(\boldsymbol{\epsilon})-A(\theta)\left(\theta-\theta_{0}\right)\right)\left(A^{\prime}(\theta)\left(\theta-\theta_{0}\right)+A(\theta)\right)+k_{\epsilon} \frac{\partial N_{c f}}{\partial \theta} \\
-\frac{\partial G\left(\theta, \boldsymbol{\epsilon}_{p}\right)}{\partial \theta} \mathbf{e}_{e} \cdot \mathbf{e}_{e}-C^{\prime}(\theta)\left[\theta \log \left(\frac{\theta}{\theta_{0}}\right)-\left(\theta-\theta_{0}\right)\right]-C(\theta) \log \left(\frac{\theta}{\theta_{0}}\right) .
\end{gathered}
$$

Similarly to elastic properties, also thermal properties depend on plastic strains through $N_{c f}$, i.e. the entropy includes an irreversible fraction, which depends on plastic strain.

Due to the presence of internal variables in the free energy density function, the internal dissipation (eqn. 5) writes

$$
\mathcal{D}_{i n t}=(\boldsymbol{\sigma}-\boldsymbol{A}) \cdot \dot{\boldsymbol{\epsilon}}_{p}+\theta \dot{\eta}_{p}
$$

where $\boldsymbol{A}=\partial \psi / \partial \boldsymbol{\epsilon}_{p}$ is the thermodynamic stress associated with $\boldsymbol{\epsilon}_{p}$. In order to obtain the maximum internal dissipation, an associated flow rule is needed with a yield function expressed as $\mathcal{F}(\boldsymbol{\sigma}-\boldsymbol{A}, \theta, \kappa)$, thus we get

$$
\dot{\boldsymbol{\epsilon}}_{p}=\dot{\Lambda} \frac{\partial \mathcal{F}(\boldsymbol{\sigma}-\boldsymbol{A}, \theta, \kappa)}{\partial(\boldsymbol{\sigma}-\boldsymbol{A})} \quad \text { and } \quad \dot{\eta}_{p}=\dot{\Lambda} \frac{\partial \mathcal{F}(\boldsymbol{\sigma}-\boldsymbol{A}, \theta, \kappa)}{\partial \theta}
$$




\subsection{Typical model response for irreversible thermal be- haviour}

Figure 3 shows the typical model response, in terms of evolution of (a) elastic stiffness, (b) fraction of closed and cured microcracks $\left(N_{c c}\right.$ and $\left.N_{c f}\right)$ and (c) longitudinal strain, along a temperature cycle. As is shown in detail in Section 7, the sample simulations of Figure 3 are consistent with the experimental trends published in the literature on a number of composite refractory materials.

It can be observed that the irreversibility of elastic stiffness and longitudinal deformation induced by temperature cycling are correctly reproduced, and thermal deformation leads to a small residual compressive strain. The evolution of closed and cured microcracks in Figure $3 \mathrm{~b}$ shows that $N_{c f}$ starts increasing along the thermal loading branch at $\mathrm{T}>800^{\circ} \mathrm{C}$ due to melting of some constituents, whereas it starts decreasing, along the unloading branch, when $\mathrm{T}<400^{\circ} \mathrm{C}$. Thus, the thermal evolution of elastic stiffness (Figure 3a) is the result of two concurrent effects: the increase of $N_{c c}$ and $N_{c f}$ leading to an increase of stiffness and the decrease of stiffness induced by melting. It can be noticed that the proposed approach has the advantage of identifying different internal variables with a clear physical meaning, characterised by evolution laws that are governed by distinct controlling variables. The proposed relationships are, in any case, fairly flexible, thus they can reliably reproduce different irreversible thermo-mechanical behaviours compared to those shown in Figure 3.

\section{Elasto-plastic coupling for thermodynamic po- tentials with irreversible thermal behaviour}

While the thermodynamic potential formulated for reversible behaviour (eqn. 9) can be considered fairly standard and self-explanatory, the free energy proposed for irreversible behaviour (eqn. 32) requires further discussion, due to the involved elasto-plastic coupling. From eqn. (37), the stress increment is equal to

$$
\dot{\boldsymbol{\sigma}}=\mathbb{D}\left[\dot{\boldsymbol{\epsilon}}_{e}\right]+\frac{\partial^{2} \psi}{\partial \boldsymbol{\epsilon}_{p} \partial \boldsymbol{\epsilon}_{e}}\left[\dot{\boldsymbol{\epsilon}}_{p}\right]-\mathbf{B} \dot{\theta},
$$

where $\mathbb{D}=\partial^{2} \psi / \partial \boldsymbol{\epsilon}_{e}^{2}$ and $\mathbf{B}=-\partial \boldsymbol{\sigma} / \partial \theta$, that can be rewritten as

$$
\dot{\boldsymbol{\sigma}}=\mathbb{D}\left[\dot{\boldsymbol{\epsilon}}-\dot{\boldsymbol{\epsilon}}_{i}\right]-\mathbf{B} \dot{\theta}
$$

where $\dot{\boldsymbol{\epsilon}}_{i}$ is the irreversible strain rate which is related to the plastic strain increment through

$$
\dot{\boldsymbol{\epsilon}}_{i}=\left(\mathbb{S}-\mathbb{D}^{-1} \frac{\partial^{2} \psi}{\partial \boldsymbol{\epsilon}_{p} \partial \boldsymbol{\epsilon}_{e}}\right)\left[\dot{\boldsymbol{\epsilon}}_{p}\right],
$$


where $\mathbb{D}$ is defined in eqn. (11), and $\mathbb{S}$ is the fourth-order tensor which singles out the symmetric part of any second-order tensor. Equation (43) is well accepted within the framework of elasto-plastic coupling (cf. [15]).

Gajo and Bigoni [7] have recently shown that the flow rule given in eqn. (40) featuring a yield function expressed as $\mathcal{F}(\boldsymbol{\sigma}-\boldsymbol{A}, \theta, \kappa)$, is equivalent to the flow rule

$$
\dot{\boldsymbol{\epsilon}}_{i}=\dot{\lambda} \frac{\partial \tilde{\mathcal{F}}(\boldsymbol{\sigma}, \theta, \tilde{\kappa})}{\partial \boldsymbol{\sigma}} \quad \text { and } \quad \dot{\eta}_{p}=\dot{\lambda} \frac{\partial \tilde{\mathcal{F}}(\boldsymbol{\sigma}, \theta, \tilde{\kappa})}{\partial \theta}
$$

with respect to a yield function in the form $\tilde{\mathcal{F}}(\boldsymbol{\sigma}, \theta, \tilde{\kappa})$.

In other terms, the flow rule can be either expressed in terms of $\dot{\boldsymbol{\epsilon}}_{p}$ which must be normal to the yield function $\mathcal{F}(\boldsymbol{\sigma}-\boldsymbol{A}, \theta, \kappa)$, or in terms of $\dot{\boldsymbol{\epsilon}}_{i}$ which must be normal to the yield function $\tilde{\mathcal{F}}(\boldsymbol{\sigma}, \theta, \tilde{\kappa})$. The two approaches are equivalent, but the latter is much simpler than the former, because the yield function $\tilde{\mathcal{F}}(\boldsymbol{\sigma}, \theta, \tilde{\kappa})$ is much easier to evaluate. This is why the latter approach will be adopted hereafter. In this case, the plastic strain increment $\dot{\boldsymbol{\epsilon}}_{p}$ must be deduced from the irreversible strain increment $\dot{\boldsymbol{\epsilon}}_{i}$, through the flow rule (eqn. 40), by using eqn. (43)

$$
\dot{\boldsymbol{\epsilon}}_{p}=\mathbb{G}^{-1}\left[\dot{\boldsymbol{\epsilon}}_{i}\right]
$$

where

$$
\mathbb{G}=\left(\mathbb{S}-\mathbb{D}^{-1} \frac{\partial^{2} \psi}{\partial \boldsymbol{\epsilon}_{p} \partial \boldsymbol{\epsilon}_{e}}\right)
$$

\section{Energy balance equation}

In the absence of heat sources, the well-known local energy balance equation (e.g., see eqn. 4-6.14 of [14]) is expressed as

$$
-\nabla \cdot \boldsymbol{q}=\dot{e}-\boldsymbol{\sigma} \cdot \dot{\boldsymbol{\epsilon}}
$$

where the symbol $\nabla$ denotes the divergence operator and $\boldsymbol{q}$ is the heat flux. If the dissipation equation (eqn. 5) is introduced in eqn. (46) and eqn. (39) is used, we obtain

$$
-\nabla \cdot \boldsymbol{q}=\theta \dot{\eta}-\mathcal{D}_{i n t}=\theta\left(\dot{\eta}-\dot{\eta}_{p}\right)-(\boldsymbol{\sigma}-\boldsymbol{A}) \cdot \dot{\boldsymbol{\epsilon}}_{p}
$$

Introducing eqn. (2) and eqn. (20) (or eqn. 38) for reversible (or irreversible) thermal behaviour, into eqn. (46), we obtain a form of the energy balance equation that does not include $\dot{\eta}_{p}$, namely

$$
-\nabla \cdot \boldsymbol{q}=\theta \dot{\eta}_{e}-(\boldsymbol{\sigma}-\boldsymbol{A}) \cdot \dot{\boldsymbol{\epsilon}}_{p}
$$

The above can be rewritten as

$$
c_{v} \dot{\theta}=(\boldsymbol{\sigma}-\boldsymbol{A}) \cdot \dot{\boldsymbol{\epsilon}}_{p}-\theta\left(\frac{\partial \boldsymbol{A}}{\partial \theta} \cdot \dot{\boldsymbol{\epsilon}}_{p}-\mathbf{B} \cdot \dot{\boldsymbol{\epsilon}}_{e}\right)-\nabla \cdot \boldsymbol{q},
$$


where $c_{v}=-\theta \partial \eta_{e} / \partial \theta$, whereas $\boldsymbol{A}=\mathbf{0}$ in the case of reversible thermal behaviour.

It is worth recalling that in metal plasticity, the mechanical dissipation $(\boldsymbol{\sigma}-$ $\boldsymbol{A}) \cdot \dot{\boldsymbol{\epsilon}}_{p}$ is typically expressed as a fraction (equal to $0.80 \div 0.95$ ) of the plastic work $\mathcal{W}_{p}=\boldsymbol{\sigma} \cdot \dot{\boldsymbol{\epsilon}}_{p}$ (see [25]). Within the proposed framework, such an empirical assumption is not needed, because all thermodynamic potentials are defined by the selected free energy function (eqn. 32). In any case, the mechanical dissipation predicted by the constitutive model must be validated against experimental evidence.

\section{Plastic driver for thermodynamic potentials with reversible and irreversible behaviour}

In this section, the procedure aimed at incorporating the above proposed thermodynamic potentials for reversible and irreversible thermal behaviour into a basic isotropic hardening yield function is described. To this aim, the following simple extension of the modified Cam Clay model [26] is considered, along the lines of existing approaches proposed for bonded geomaterials ([9], [22], [23]), where the set of internal variables (denoted with $\kappa$ in eqn. 26 , and with $\tilde{\kappa}$ in eqn. 44 ) is set equal to the plastic volumetric strain (i.e. $\left.\operatorname{Tr} \boldsymbol{\epsilon}_{p}\right)$ :

$$
\mathcal{F}\left(\boldsymbol{\sigma}, \theta, \operatorname{Tr} \boldsymbol{\epsilon}_{p}\right)=M_{c v} K_{\theta}\left(\left(\frac{\operatorname{Tr}(\boldsymbol{\sigma})}{3}-P_{t}\right)^{2}+\left(\frac{\operatorname{Tr}(\boldsymbol{\sigma})}{3}-P_{t r}\right)\left(P_{c}+P_{t}\right)\right)+3 J_{2}
$$

where $M_{c v}$ is the critical state slope parameter, $K_{\theta}$ is a function of the Lode angle describing the deviatoric section of the yield surface (in this work the function proposed by Willam and Warnke [33] has been used), $J_{2}$ is the second stress invariant, $P_{t}$ and $P_{c}$ are the tensile and compressive strengths of the material. $P_{t}$ and $P_{c}$ are assumed related to the number of cured fractures through

$$
P_{t}=N_{c f} \sigma_{r t} \quad \text { and } \quad P_{c}=P_{0} \operatorname{Exp}\left(-\frac{\operatorname{Tr} \epsilon_{p}+\epsilon_{0}}{\lambda}\right)+\left(N_{c c}+N_{c f}\right) \sigma_{r c}
$$

where $\lambda$ is a constitutive parameter, $P_{0}$ and $\epsilon_{0}$ are the reference mean pressure and volumetric strain, and $\sigma_{r t}(\theta)$ and $\sigma_{r c}(\theta)$ are the tensile and compressive strength at the microscopic level, depending on temperature $\theta$. The number of closed and cured cracks, $N_{c c}$ and $N_{c f}$, is evaluated by using eqns. (28) and (29). When the material is completely uncemented, $N_{c f}=0$, then the tensile strength is null $P_{t}=0$, and $P_{c}$ becomes equal to the preconsolidation pressure of a loose (uncemented) geomaterial plus a contribution due to closed microcracks, namely

$$
P_{c}=P_{0} \operatorname{Exp}\left(-\frac{\operatorname{Tr} \epsilon_{p}+\epsilon_{0}}{\lambda}\right)+N_{c c} \sigma_{r c}
$$


The first term of the above coincides with the typical Cam Clay hardening relationship (e.g., see [21]).

As an example, the following tentative dependency of $\sigma_{r t}$ and $\sigma_{r c}$ on temperature can be assumed

$$
\begin{gathered}
\sigma_{r c}=a_{R C}-b_{R C} \operatorname{Tanh}\left(\frac{\theta-\theta_{M}}{r_{R C}}\right), \\
\sigma_{r t}=\frac{\sigma_{r c}}{r_{f}}
\end{gathered}
$$

where $a_{R C}, b_{R C}$ and $r_{R C}$ are constitutive parameters and $r_{f}$ is the ratio between the tensile and compressive strength at microscopic level. Note that tensile and compressive strengths at the microscopic level, following common engineering sense, are assumed to decrease with the increase of temperature, following a trend similar to the variation of elastic stiffness of alumina (eqn. 21).

Figure $3 \mathrm{~d}$ shows the simulated variation of tensile and compressive strengths $P_{c}$ and $P_{t}$ versus temperature. It is worth observing that due to the irreversibility of the thermal dependency of $N_{c f}$, if a thermal unloading branch is followed, $P_{c}$ and $P_{t}$ are larger compared to the values they take at the beginning of a thermal loading branch. In fact, upon thermal unloading $P_{c}$ and $P_{t}$ start decreasing at lower temperatures, due to microcracks opening resulting from the differential thermal expansion of the constituents.

For the sake of simplicity, the flow rule is assumed associated.

\subsection{Tangent operator in the case of reversible thermal be- haviour}

From the free energy density function (eqn. 9), the stress rate and the entropy rate are obtained as

$$
\begin{aligned}
\dot{\boldsymbol{\sigma}} & =\mathbb{D}\left[\dot{\boldsymbol{\epsilon}}_{e}\right]-\mathbf{B} \dot{\theta} \\
\dot{\eta}_{e} & =\mathbf{B} \cdot \dot{\boldsymbol{\epsilon}}_{e}-\frac{c_{v}}{\theta} \dot{\theta}
\end{aligned}
$$

where $c_{v}=-\theta \partial \eta_{e} / \partial \theta$ and $\mathbf{B}=-\partial \boldsymbol{\sigma} / \partial \theta=\partial \eta_{e} / \partial \boldsymbol{\epsilon}_{e}=-\partial^{2} \psi / \partial \boldsymbol{\epsilon}_{e} \partial \theta$.

Denoting the yield function gradient with $\mathbf{Q}=\partial \mathcal{F} / \partial \boldsymbol{\sigma}$, the plastic flow direction with $\mathbf{P}(\mathbf{P}=\mathbf{Q}$ in this case, due to the assumption of associated flow rule) and the hardening parameter with $H=-\left(\partial \mathcal{F} / \partial \operatorname{Tr} \boldsymbol{\epsilon}_{p}\right) \operatorname{Tr} \mathbf{P}$, the consistency condition $(\dot{\mathcal{F}}=0)$ yields

$$
\dot{\Lambda}=\frac{<\mathbf{Q} \cdot(\mathbb{D}[\dot{\boldsymbol{\epsilon}}]-\mathbf{B} \dot{\theta})+(\partial \mathcal{F} / \partial \theta) \dot{\theta}>}{\mathbf{Q} \cdot \mathbb{D}[\mathbf{P}]+H}
$$


Thus, in case of plastic loading (corresponding to a positive argument within the Macaulay brackets in eqn. 57), the tangent constitutive operator results in

$$
\begin{gathered}
\dot{\boldsymbol{\sigma}}=\left\{\mathbb{D}-\frac{\mathbb{D}[\mathbf{P}] \otimes \mathbb{D}[\mathbf{Q}]}{\mathbf{Q} \cdot \mathbb{D}[\mathbf{P}]+H}\right\}[\dot{\boldsymbol{\epsilon}}]-\left(\mathbf{B}-\frac{\mathbf{B} \cdot \mathbf{Q}-(\partial \mathcal{F} / \partial \theta)}{\mathbf{Q} \cdot \mathbb{D}[\mathbf{P}]+H} \mathbb{D}[\mathbf{P}]\right) \dot{\theta} \\
\dot{\eta}_{e}=\left(\mathbf{B}-\frac{\mathbf{B} \cdot \mathbf{P}}{\mathbf{Q} \cdot \mathbb{D}[\mathbf{P}]+H} \mathbb{D}[\mathbf{Q}]\right) \cdot \dot{\boldsymbol{\epsilon}}+\left(\frac{\mathbf{B} \cdot \mathbf{Q}-(\partial \mathcal{F} / \partial \theta)}{\mathbf{Q} \cdot \mathbb{D}[\mathbf{P}]+H} \mathbf{B} \cdot \mathbf{P}-\frac{c_{v}}{\theta}\right) \dot{\theta}
\end{gathered}
$$

It emerges from the above equations that the tangent operator is not symmetric even in the case of associated flow rule (i.e. $\mathbf{P}=\mathbf{Q}$ ) and of a yield surface independent of temperature (i.e. $\partial \mathcal{F} / \partial \theta=0$ ). However, if instead of the elastic part $\dot{\eta}_{e}$ the total entropy rate $\dot{\eta}=\dot{\eta}_{e}+\dot{\eta}_{p}$ was considered, the corresponding tangent operator would be symmetric.

\subsection{Tangent operator in the case of irreversible thermal behaviour}

From the free energy density function (eqn. 32), the stress and entropy rates are obtained as

$$
\begin{gathered}
\dot{\boldsymbol{\sigma}}=\mathbb{D}\left[\dot{\boldsymbol{\epsilon}}-\dot{\boldsymbol{\epsilon}}_{i}\right]-\mathbf{B} \dot{\theta} \\
\dot{\eta}_{e}=\mathbf{B} \cdot \dot{\boldsymbol{\epsilon}}+(\mathbf{T}-\mathbf{B}) \dot{\boldsymbol{\epsilon}}_{p}-\frac{c_{v}}{\theta} \dot{\theta}
\end{gathered}
$$

where $\mathbf{T}=\partial \eta_{e} / \partial \boldsymbol{\epsilon}_{p}=-\partial \boldsymbol{A} / \partial \theta$.

Taking account of eqn. (45), the consistency condition yields

$$
\dot{\Lambda}=\frac{<\mathbf{Q} \cdot(\mathbb{D}[\dot{\boldsymbol{\epsilon}}]-\mathbf{B} \dot{\theta})+(\partial \mathcal{F} / \partial \theta) \dot{\theta}>}{\mathbf{Q} \cdot \mathbb{D}[\mathbf{P}]+\tilde{H}}
$$

where $\tilde{H}=-\left(\partial \mathcal{F} / \partial \operatorname{Tr} \boldsymbol{\epsilon}_{p}\right) \operatorname{Tr}\left(\mathbb{G}^{-1}[\mathbf{P}]\right)$.

As a result, in case of plastic loading (corresponding to a positive argument within the Macaulay brackets in eqn. 62), the tangent constitutive operator turns out to be

$$
\begin{gathered}
\dot{\boldsymbol{\sigma}}=\left\{\mathbb{D}-\frac{\mathbb{D}[\mathbf{P}] \otimes \mathbb{D}[\mathbf{Q}]}{\mathbf{Q} \cdot \mathbb{D}[\mathbf{P}]+\tilde{H}}\right\}[\dot{\boldsymbol{\epsilon}}]-\left(\mathbf{B}-\frac{\mathbf{B} \cdot \mathbf{Q}-(\partial \mathcal{F} / \partial \theta)}{\mathbf{Q} \cdot \mathbb{D}[\mathbf{P}]+\tilde{H}} \mathbb{D}[\mathbf{P}]\right) \dot{\theta} \\
\dot{\eta}_{e}=\left(\mathbf{B}+\frac{(\mathbf{T}-\mathbf{B}) \cdot\left(\mathbb{G}^{-1}[\mathbf{P}]\right)}{\mathbf{Q} \cdot \mathbb{D}[\mathbf{P}]+\tilde{H}} \mathbb{D}[\mathbf{Q}]\right) \cdot \dot{\boldsymbol{\epsilon}}+ \\
-\left(\frac{\mathbf{B} \cdot \mathbf{Q}-(\partial \mathcal{F} / \partial \theta)}{\mathbf{Q} \cdot \mathbb{D}[\mathbf{P}]+\tilde{H}}(\mathbf{T}-\mathbf{B}) \cdot\left(\mathbb{G}^{-1}[\mathbf{P}]\right)-\frac{c_{v}}{\theta}\right) \dot{\theta} .
\end{gathered}
$$

Note that the tangent operator is not symmetric even in the case of associated flow rule (i.e. $\mathbf{P}=\mathbf{Q}$ ), yield surface independent of temperature (i.e. $\partial \mathcal{F} / \partial \theta=0$ ) 
and absent elastoplastic coupling (i.e. $\mathbb{G}=\mathbb{I}$, where $\mathbb{I}$ denotes the fourth order identity tensor, and $\mathbf{T}=\mathbf{0}$ ). If instead of the elastic part $\dot{\eta}_{e}$ the total entropy rate $\dot{\eta}$ was considered, the corresponding tangent operator would be symmetric.

\section{Model validation}

In order to validate the model capabilities to reproduce the experimental thermomechanical behaviour of different types of refractories, a series of experimental loading paths were selected from the literature. Model parameters were chosen based on the available literature information, and specifically calibrated on each of the selected experimental datasets. The main adopted parameter values are summarized in Table 1.

It should be remarked that although the total number of model parameters may be considered rather large, the wide majority of constants reported in Table 1 are merely curve fitting parameters (as it emerges from the formulation reported in Sections 2 and 3), thus they can be straightforwardly determined upon availability of experimental data. A basic set of tests that is desirable for model calibration is composed of measurements at atmospheric pressure of the thermal dependency of elasitc modulus, specific heat and thermal expansion for thermoelasticity, and of uniaxial compressive and tensile tests for thermo-plasticity.

Special attention for model validation was given to the available experiments on composite refractories, whose behaviour is known to exhibit a high degree of nonlinearity and irreversibility. The observed experimental trends were reproduced by numerically integrating the thermo-elasto-plastic model equations (Sections 3-6) through an implicit backward Euler scheme.

\subsection{Evolution of elastic properties under thermal cycles of different amplitude}

First, the experiments carried out by Kakroudi et al. [10] on an ultra low cement commercial Bauxite based refractory castable were considered, involving the ultrasonic measurement of Young's modulus of elasticty $E$ at different temperature levels during thermal loading-unloading cycles. The main adopted parameter values for this material are summarized in Table 1 (Set 2) and initial conditions are reported in Table 2 (Set 2). In Figure 4a, the evolution of elastic modulus is reproduced numerically during four thermal cycles, conducted from ambient temperature up to four different maximum temperatures, namely 700, 900, 1100 and $1500^{\circ} \mathrm{C}$. This type of testing highlights the presence of different stages in the evolution of $E$, with particular reference to a threshold temperature (between 700 and $900^{\circ} \mathrm{C}$ in this case) below which the heating-cooling path appears mostly

reversible. A second threshold corresponds to about $1200^{\circ} \mathrm{C}$, beyond which the elastic modulus starts to drastically decrease with further increasing temperature, 
in correspondence with the likely appearence of low-viscosity, glassy phases. In Figure $4 \mathrm{~b}$ the corresponding experimental data from [10] are reported for comparison. In addition, in Figure 4c the simulated thermally induced axial strain $d L / L_{0}$, whose slope represents the thermal expansion coefficient of the material, is reported versus temperature for the four thermal cycles described above, the wider of which reaches $1500^{\circ} \mathrm{C}$. The simulations appear to compare well with the relevant experimental data by [10], reported in Figure 4d (only data from the $20-1500^{\circ} \mathrm{C}$ experimental cycle are available).

Moreover, in Figures 5a and 5b the simulated and experimentally measured evolution of $E$ are respectively shown, during two whole thermal cycles between ambient temperature and $1200^{\circ} \mathrm{C}$ for the same Bauxite based refractory, tested by Kakroudi et al. [10]. It can be observed that the model is able to capture with reasonable accuracy the experimental trend also during the second thermal cycle, which exhibits a fairly smaller amount of hysteresis compared to the first one.

\subsection{Evolution of unconfined compressive and tensile strength upon thermal loading}

In addition to ultrasonic measurements, Kakroudi et al. [10] also carried out hightemperature tensile tests on the same material. These were performed always at $800^{\circ} \mathrm{C}$, but in three different points of the thermal cycling history, namely (i) during the first loading branch (point $\mathrm{A}$ in Figure 5b), (ii) during the first unloading branch (point $\mathrm{B}$ in Figure 5b) and (iii) during the second loading branch (point $\mathrm{C}$ in Figure 5b). In Figure $5 \mathrm{c}$ and Figure $5 \mathrm{~d}$ the experimental data and corresponding numerical simulations are shown, respectively. Even though the specific form of yield surface chosen typically does not allow to reproduce particularly pronounced post-peak strain softening behaviour, it can be noticed that both the changes in elastic stiffness and in the maximum tensile strength are correctly captured by the model.

The model's predictive capabilities were further tested against experimental data of uniaxial compression tests carried out at different temperatures on unfired samples of alumina-carbon refractory concrete, presented by Ouedraogo and Prompt [24]. The parameter settings and initial conditions for this material are listed in Table 1 (Set 3) and Table 2 (Set 3) respectively. In Figure 6a, the simulated and measured evolution of uniaxial compressive strength (UCS) with temperature are reported for comparison, with temperature ranging from 20 to $1500^{\circ} \mathrm{C}$. Despite the rather complex, highly nonlinear trend followed by experimental datapoints, the model is shown to capture properly the essential features of thermal dependency of uniaxial compressive strength of composite refractory materials. The same can be also observed in terms of tensile strength, as the Brazilian test results obtained by [24] at different temperatures are properly 
reproduced by simulations, as shown in Figure $6 \mathrm{~b}$.

\subsection{Elastic stiffness degradation upon cyclic loading during tensile testing}

Finally, the model was employed to simulate the evolution of damage during tensile testing at room temperature. In this case, reference is made to the experiments on magnesia-spinel refractory samples reported by Grasset-Bourdel et al. [12]. Relevant parameter settings and initial conditions correspond to Set 4 of Table 1 and Table 2 respectively. In Figure 7, simulations are compared to experimental data of tensile tests carried out with several mechanical unloadingreloading cycles, both before and after the peak strength. It can be observed that the numerical simulations can adequately reproduce the overall strain-softening trend of the stress-strain material behaviour. Moreover, although the experimentally observed hysteresis during the unloading-reloading cycles is not reproduced by the model, the mechanical damage in terms of progressive increase of slope, i.e. a reduction in elastic stiffness with an increase of plastic straining, is correctly captured.

\section{Conclusions}

In this work a general, thermodynamically consistent constitutive framework, able to reproduce the non isothermal behaviour of rock-like materials subjected to very high temperature, is developed and validated against experimental data. Particular attention is given to ceramic refractories employed in the steel making industry, for which experimental data availability is relatively wide. However, these materials show different thermo-mechanical trends depending upon their composition, in that single component refractories tend to exhibit reversible behaviour, while composite refractories usually exhibit irreversibility, upon thermal cycling. Thus, two distinct forms of thermodynamic potentials, that constitute the basis for subsequent derivation of constitutive relationships, are proposed to reproduce reversible and irreversible behaviour. A key advantage of this approach is the ability to freely choose the interpolation functions representing the thermal dependency of thermo-elastic material parameters, hence even complex experimental trends can be relatively easily captured upon calibrating the interpolation parameters.

Firstly, to validate the model, the (reversible) experimentally measured thermal dependency of elastic stiffness, specific heat and thermal expansion of sintered alumina are numerically reproduced. Further, the model allows exploring loading conditions that are not usually considered, or viable, in the experimental practice. In fact, while a sample of alumina subjected to low confining stress exhibits negligible thermo-mechanical coupling, if it is subject to an applied stress 
level comparable to its stiffness, the material response becomes coupled, and unexpected trends occur in the material's specific heat and thermal expansion vs temperature curves.

To reproduce the irreversible thermo-mechanical behaviour exhibited by composite materials, thermodynamic potentials are set to include internal variables representing microstructural effects, namely the amount of closed microcracks due to isotropic volumetric strain and the amount of cured microcracks due to partial melting. The constitutive framework in this case is more complex, and is shown to imply elasto-plastic coupling. Moreover, a plastic driver incorporating both types of thermodynamic potentials is formulated, and employed for extensive model validation against thermo-mechanical experimental datapoints on composite refractories. The highly nonlinear and hysteretic trends exhibited experimentally are correctly reproduced under several types of loading conditions, including temperature cycling and uniaxial compressive and tensile testing, thus demonstrating the robustness of the proposed approach.

A possible extension of the proposed model could be a formulation in terms of finite strains, to provide more accurate simulations of the irreversible phenomena occurring in certain applications. This could be done along the lines of existing approaches, as those proposed in (e.g.) [28], [30], [18], [19], [4], [5], and [6].

To conclude, the proposed general framework can be successfully applied to model the key features of the behaviour of different types of refractories under a number of thermo-mechanical loading conditions. However, given its flexibility and generality, the model should be intended as an open framework that can be easily adapted to other geomaterial types, or to incorporate different additional effects.

Acknowledgements: Financial support form the EU research project PIAPPGA-2013-609758-HOTBRICKS is gratefully acknowledged.

\section{References}

[1] A Benallal and D Bigoni. Effects of temperature and thermo-mechanical couplings on material instabilities and strain localization of inelastic materials. Journal of the Mechanics and Physics of Solids, 52(3):725-753, 2004.

[2] F Cecinato, A Zervos, and E Veveakis. A thermo-mechanical model for the catastrophic collapse of large landslides. International Journal for Numerical and Analytical Methods in Geomechanics, 35(14):1507-1535, 2011.

[3] B D Coleman and W Noll. The thermodynamics of elastic materials with heat conduction and viscosity. Archive for Rational Mechanics and Analysis, 13(1):167-178, 1963. 
[4] A Gajo. A general approach to isothermal hyperelastic modelling of saturated porous media at finite strains with compressible solid constituents. In Proceedings of the Royal Society of London A: Mathematical, Physical and Engineering Sciences, page rspa20100018. The Royal Society, 2010.

[5] A Gajo. Finite strain hyperelastoplastic modelling of saturated porous media with compressible constituents. International Journal of Solids and Structures, 48(11):1738-1753, 2011.

[6] A Gajo. A general approach for defining the macroscopic free energy density of saturated porous media at finite strains under non-isothermal conditions. European Journal of Mechanics-A/Solids, 30(6):1040-1054, 2011.

[7] A Gajo and D Bigoni. Elastoplastic coupling for thermo-elasto-plasticity at high temperature. Geomechanics for Energy and the Environment, 2015, in press.

[8] A Gajo and F Cecinato. Some considerations about thermomechanical and thermophysical modelling of ceramic refractory materials. In "State of the art and challenges in thermal and mechanical modelling of ceramic materials" Workshop, Trento, 2015.

[9] A Gens and R Nova. Conceptual bases for a constitutive model for bonded soils and weak rocks. Geotechnical engineering of hard soils-soft rocks, 1(1):485-494, 1993.

[10] M Ghassemi Kakroudi, E Yeugo-Fogaing, M Huger, C Gault, and T Chotard. Influence of the thermal history on the mechanical properties of two alumina based castables. Journal of the European Ceramic Society, 29(15):3197-3204, 2009.

[11] P Goetze, R Wulf, U Gross, M Dopita, D Rafaja, S Dudczig, C G Aneziris, Y Klemm, and H B Biermann. Thermophysical properties of pressed and casted carbon-bonded alumina (al2o3-c) up to $800{ }^{\circ} \mathrm{c}$. Advanced Engineering Materials, 15(12):1270-1279, 2013.

[12] R Grasset-Bourdel, A Alzina, M Huger, D Gruber, H Harmuth, and $\mathrm{T}$ Chotard. Influence of thermal damage occurrence at microstructural scale on the thermomechanical behaviour of magnesia-spinel refractories. Journal of the European Ceramic Society, 32(5):989-999, 2012.

[13] A E Green and P M Naghdi. Thermoelasticity without energy dissipation. Journal of elasticity, 31(3):189-208, 1993.

[14] R Haase. Thermodynamics of irreversible processes. Dover Publications, 1990. 
[15] T Hueckel and G Maier. Incremental boundary value problems in the presence of coupling of elastic and plastic deformations: a rock mechanics oriented theory. International Journal of Solids and Structures, 13(1):1-15, 1977.

[16] M Huger, C Gault, and T Chotard. High temperature elastic properties of refractory materials. Iranian Journal of Materials Science and Engineering, $4(3): 44-52,2007$.

[17] Y Joliff, J Absi, M Huger, and J C Glandus. Experimental and numerical study of the elastic modulus vs temperature of debonded model materials. Computational Materials Science, 44(2):826-831, 2008.

[18] L Lanzoni and A M Tarantino. Damaged hyperelastic membranes. International Journal of Non-Linear Mechanics, 60:9-22, 2014.

[19] L Lanzoni and A M Tarantino. Equilibrium configurations and stability of a damaged body under uniaxial tractions. Zeitschrift für angewandte Mathematik und Physik, 66(1):171-190, 2015.

[20] F Marotti de Sciarra and M Salerno. On thermodynamic functions in thermoelasticity without energy dissipation. European Journal of MechanicsA/Solids, 46:84-95, 2014.

[21] D Muir Wood. Soil behaviour and critical state soil mechanics. Cambridge university press, 1990.

[22] R Nova. Modelling weathering effects on the mechanical behaviour of granite. In Constitutive modelling of granular materials, pages 397-411. Springer, 2000 .

[23] R Nova, R Castellanza, and C Tamagnini. A constitutive model for bonded geomaterials subject to mechanical and/or chemical degradation. International Journal for Numerical and Analytical Methods in Geomechanics, 27(9):705-732, 2003.

[24] E Ouedraogo and N Prompt. High-temperature mechanical characterisation of an alumina refractory concrete for blast furnace main trough: Part ii. material behaviour. Journal of the European Ceramic Society, 28(15):2867$2875,2008$.

[25] M Ristinmaa, M Wallin, and N S Ottosen. Thermodynamic format and heat generation of isotropic hardening plasticity. Acta mechanica, 194(1-4):103$121,2007$.

[26] K H Roscoe and J B Burland. On the generalized stress-strain behaviour of wet clay. In Engineering Plasticity. Cambridge University Press, 1968. 
[27] J C Simo and C Miehe. Associated coupled thermoplasticity at finite strains: Formulation, numerical analysis and implementation. Comput. Methods Appl. Mech. Eng., 98(1):41-104, July 1992.

[28] S Stupkiewicz, A Piccolroaz, and D Bigoni. Finite-strain formulation and fe implementation of a constitutive model for powder compaction. Computer Methods in Applied Mechanics and Engineering, 283:856-880, 2015.

[29] J Sulem, P Lazar, and I Vardoulakis. Thermo-poro-mechanical properties of clayey gouge and application to rapid fault shearing. International journal for numerical and analytical methods in geomechanics, 31(3):523-540, 2007.

[30] A M Tarantino. Equilibrium paths of a hyperelastic body under progressive damage. Journal of Elasticity, 114(2):225-250, 2014.

[31] N Tessier-Doyen, J C Glandus, and M Huger. Untypical young's modulus evolution of model refractories at high temperature. Journal of the European Ceramic Society, 26(3):289-295, 2006.

[32] J Werner, C G Aneziris, and S Dudczig. Young's modulus of elasticity of carbon-bonded alumina materials up to $1450{ }^{\circ} \mathrm{c}$. Journal of the American Ceramic Society, 96(9):2958-2965, 2013.

[33] K J William and E P Warnke. Constitutive models for the triaxial behavior of concrete. Proceedings of the International Assoc. for Bridge and Structural Engineering, 19:1-30, 1975. 

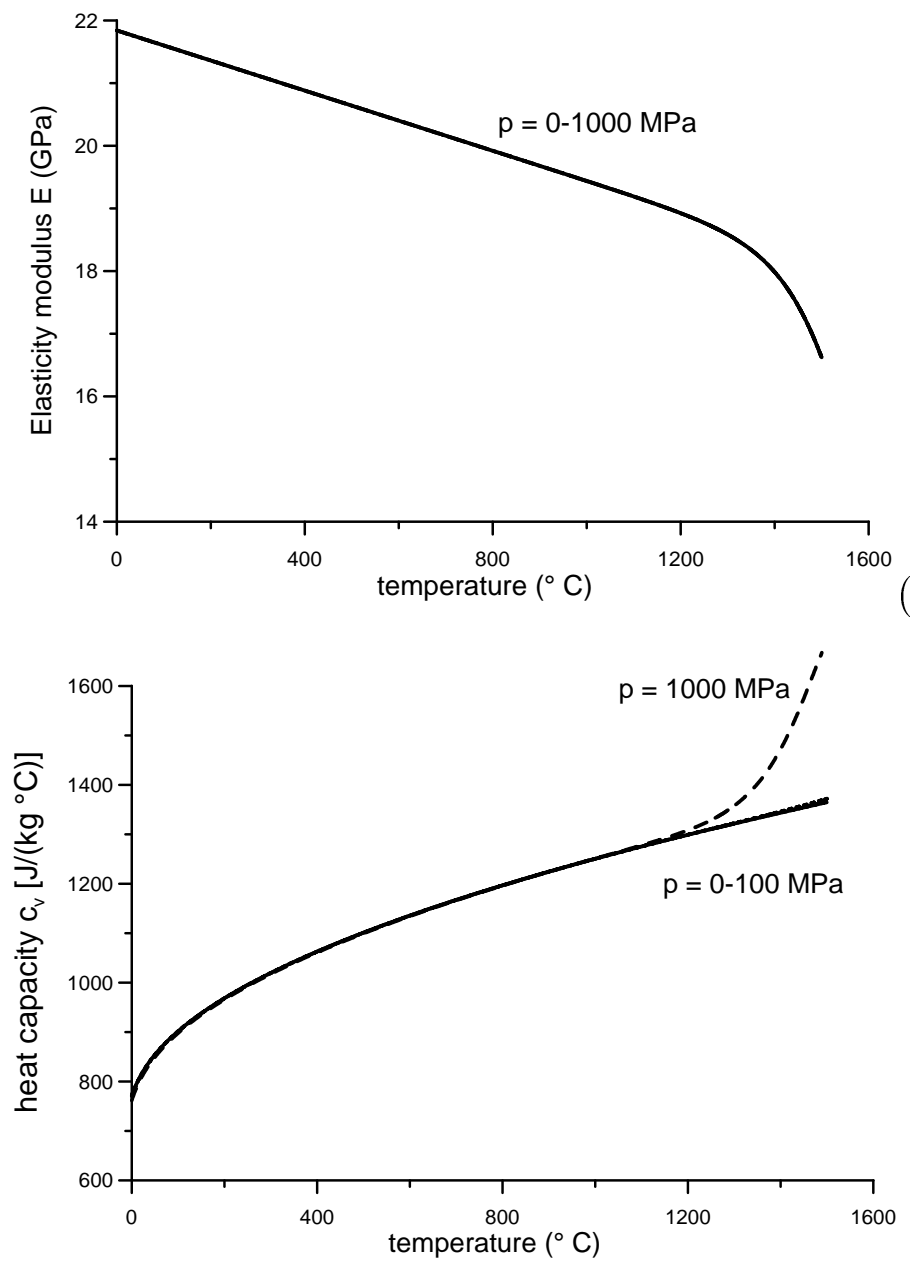

(a)

(b)

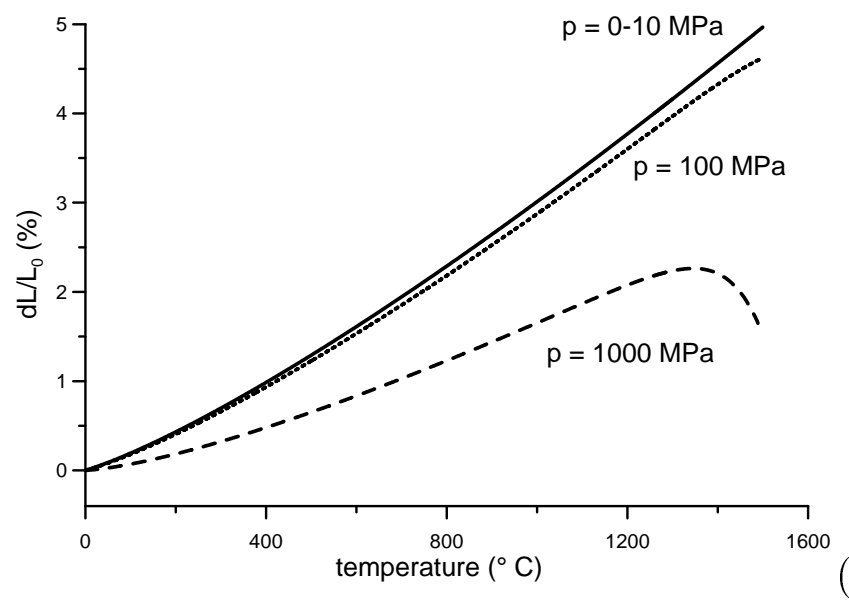

(c)

Figure 1: Temperature dependency of (a) elastic stiffness, (b) specific heat at constant volume and (c) longitudinal deformations, in the case of reversible thermal behaviour (alumina). 


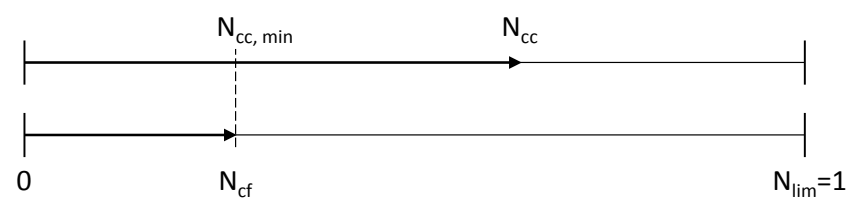

Figure 2: Schematic illustrating the ranges of variation of the two internal variables $N_{c c}$ and $N_{c f}$.
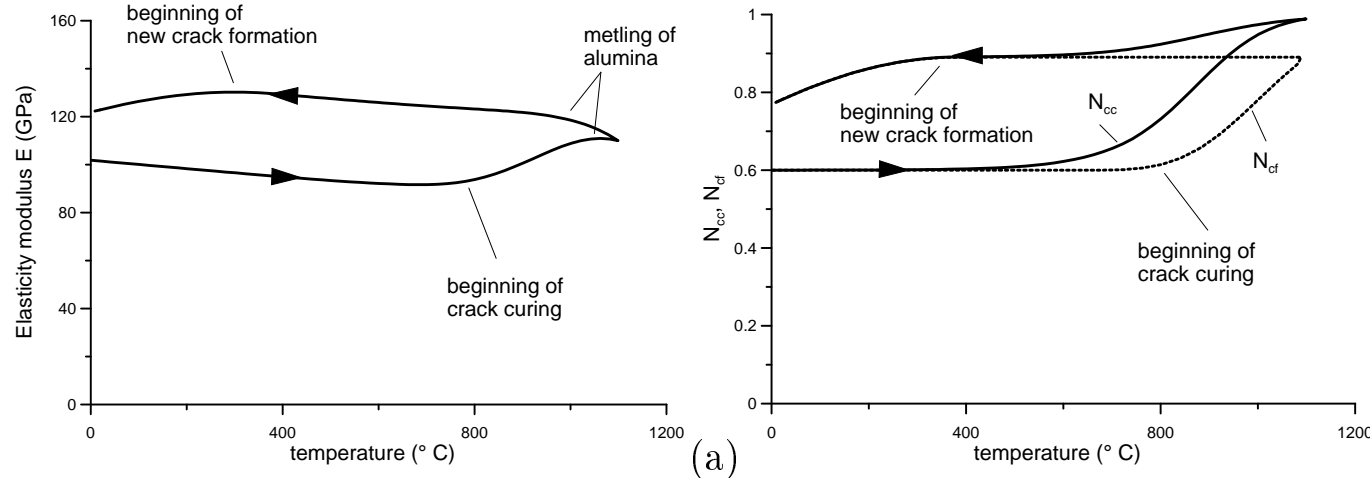

(b)
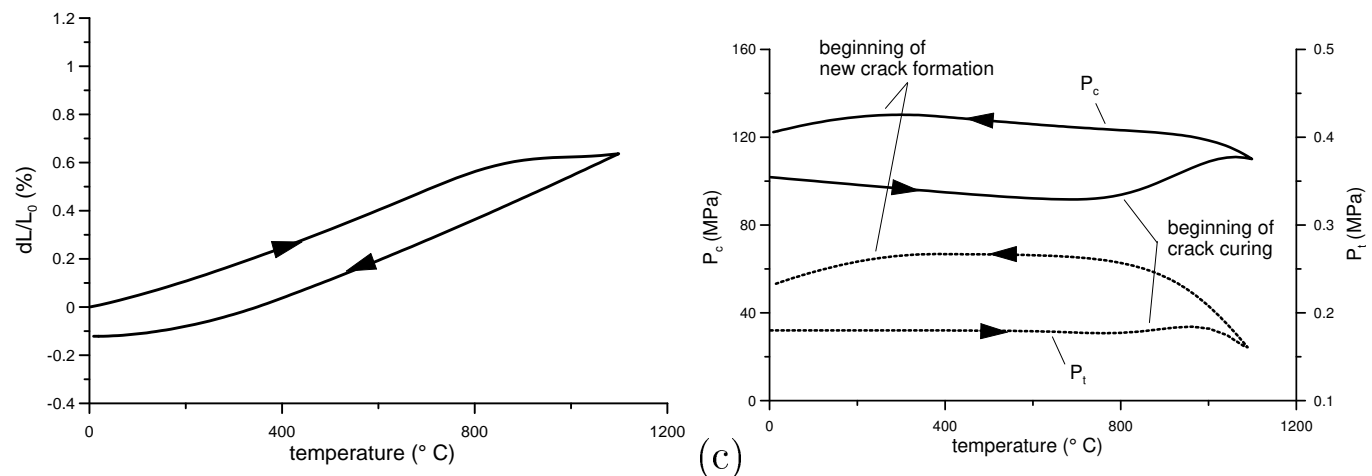

(d)

Figure 3: Temperature dependence of (a) elastic stiffness, (b) fraction of closed and cured cracks $\left(N_{c c}\right.$ and $\left.N_{c f}\right),(\mathrm{c})$ longitudinal deformations, in the case of irreversible thermal behaviour (composite refractories), and (d) material tensile and compressive strength. 

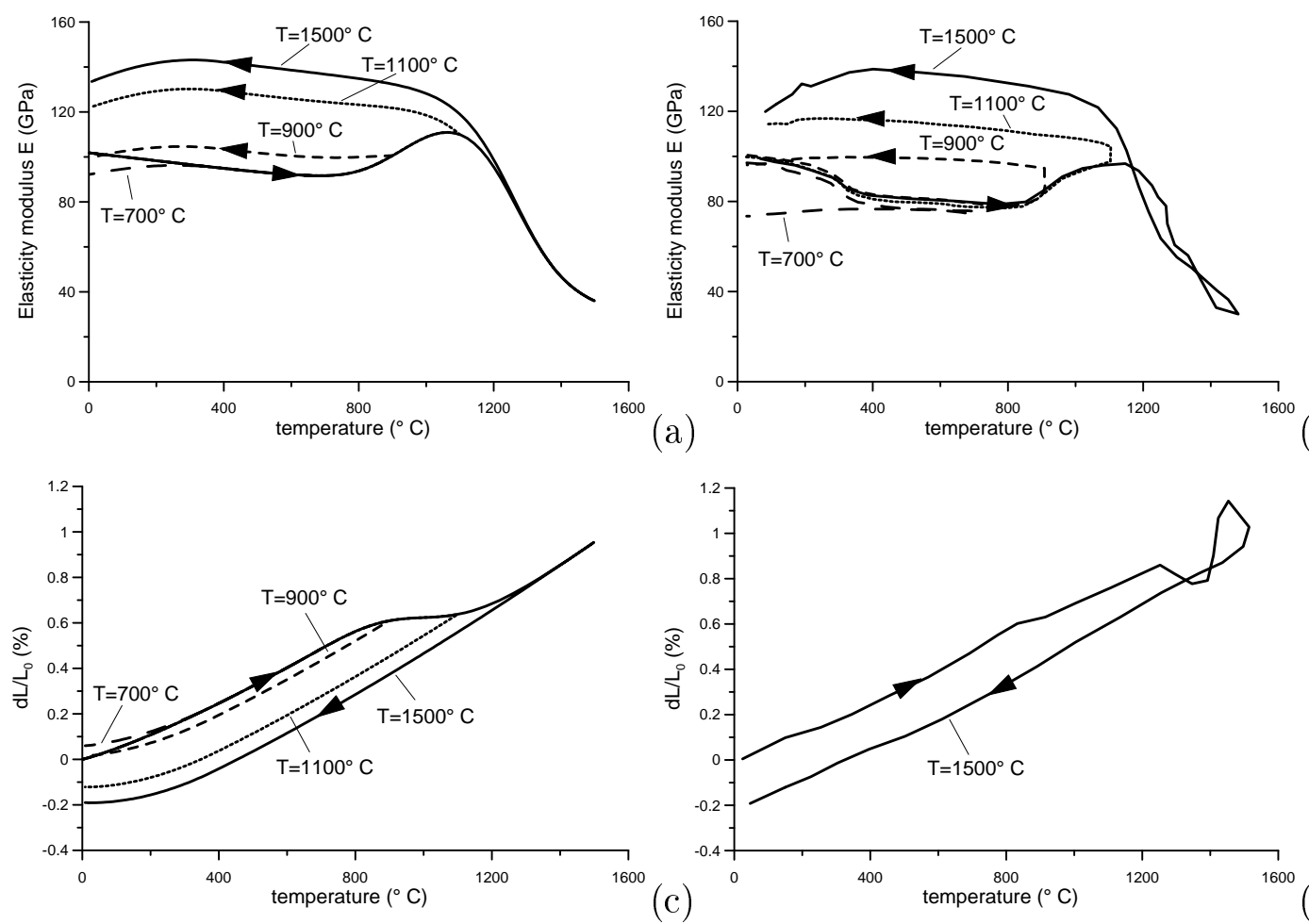

Figure 4: Temperature dependence of elastic stiffness and longitudinal deformation induced by temeprature cycles of different amplitude. Simulations ((a) and (c)) are compared with the experimental results of Kakroudi et al. [10] on a composite refractory ((b) and (d)) 

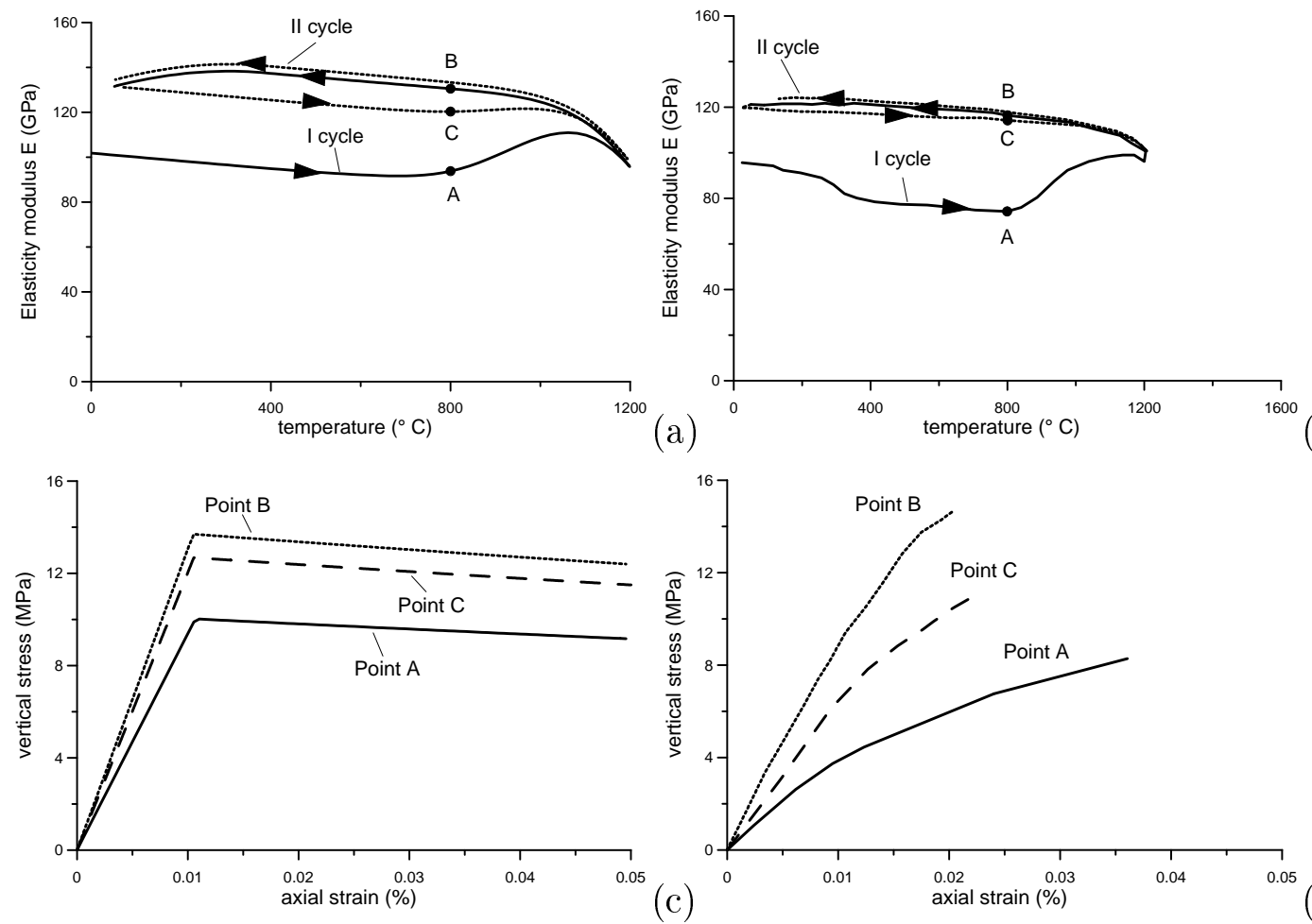

Figure 5: Evolution of elastic stiffness and tensile strength along two subsequent temeprature cycles of equal amplitude $\left(0-1200^{\circ} \mathrm{C}\right)$. Simulations ((a) and (c)) are compared with the experimental results of Kakroudi et al. [10] on a composite refractory ((b) and (d)).
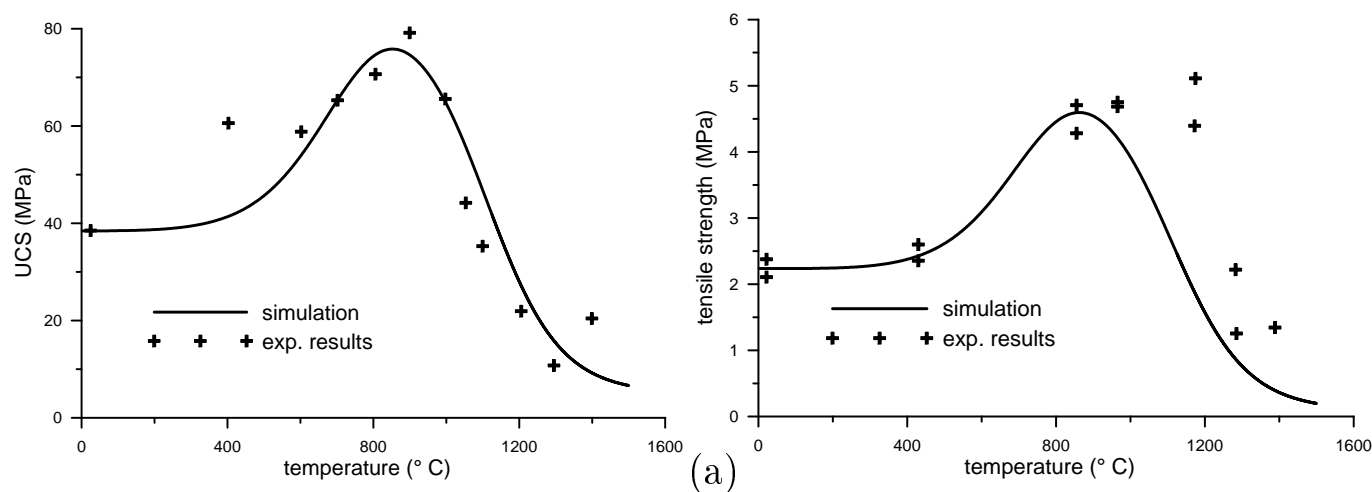

(b)

Figure 6: Temperature dependence of unconfined compression (a) and tensile (b) strengths: comparison between simulations and experimental results on a composite refractory. Experimental data are taken from [24]. 


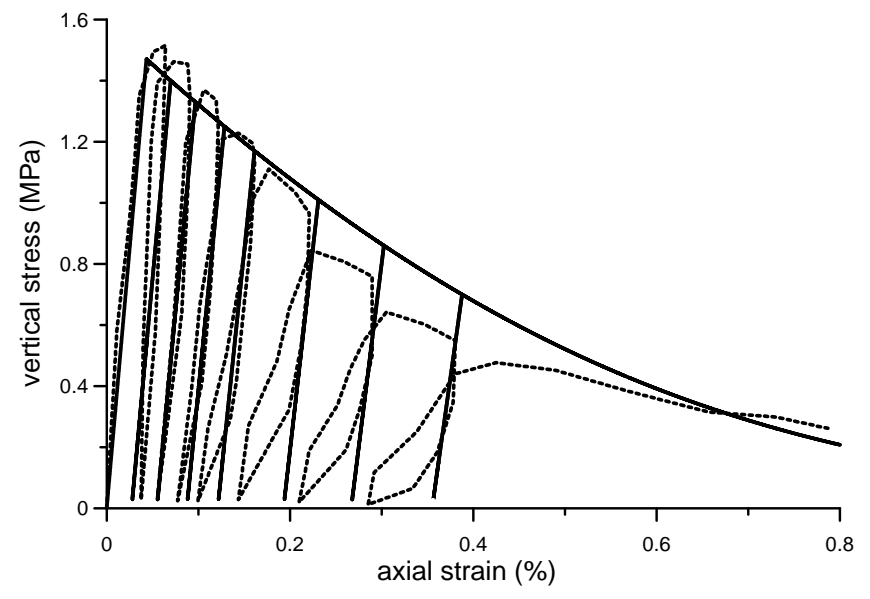

Figure 7: Comparison between simulation (solid line) and experimental results (dotted line) of a cyclic tensile test on a composite refractory. Experimental data are taken from [12]. 